\title{
EFFICIENCY RENTS OF PUMPED-STORAGE PLANTS AND THEIR USES FOR OPERATION AND INVESTMENT DECISIONS
}

\author{
by \\ Anthony Horsley and Andrew J Wrobel \\ London School of Economics and Political Science
}

Contents:

Abstract

1. Introduction

2. Pumped-Storage Technology

3. Heuristics for Valuation of Stock and Capacities

Figure 1

4. The Linear Programme of Plant Operation

5. Capacity Valuation as the Dual Linear Programme

6. Conditions for Optimal Operation and Valuation

7. Shadow Pricing of Stock as the Dual Problem

8. Determination of Optimal Storage Policy

9. Marginal Capacity Values in Terms of Stock Price

10. Bounds on Marginal Capacity Values

11. Optimum Investment in Storage Plants

Figures $2 \mathrm{a}$ and $2 \mathrm{~b}$

12. Indeterminacy of Marginal Values with Discrete Time

13. Conclusions

Appendix A. Proofs

References

Discussion Paper

No.TE/00/405

November 2000
The Suntory Centre

Suntory and Toyota International Centres for Economics and Related Disciplines London School of Economics and Political Science

Houghton Street

London WC2A 2AE

Tel.: 020- 74057686 


\begin{abstract}
We apply duality methods of linear and convex programming to the problems of operation and rental valuation of facilities for conversion and storage of cyclically priced goods, e.g. , energy. Both problems are approached by shadow-pricing the stock (which is a purely intermediate commodity); and if the given market price $p$ for the final good is a continuous function of time, then the stock's shadow price function $\psi$ is shown to be unique (and continuous). Therefore, despite being perfect Allen-Hicks complements, the plant's capacities have definite and separate marginal values, which are expressed in terms of $\psi$ (and $p$ ). In particular, the unit reservoir rent equals the total positive variation of $\psi$ over the cycle. The optimal storage policy is also given in terms of $\psi$ and $p$ ). The marginal capacity values are used to determine the optimum investment. The framework can accommodate related storage problems (such as hydroelectric generation).
\end{abstract}

Keywords: Pumped storage; peak-load pricing; marginal values; linear programming.

JEL Nos.: C61, D24, D46, L94, L95.

(C) by the authors. All rights reserved. Short sections of text, not to exceed two paragraphs, may be quoted without explicit permission provided that full credit, including (C) notice, is given to the source. 


\section{INTRODUCTION}

The problems of optimal operation and rental valuation of storage facilities for cyclically priced goods have been studied mainly in the context of hydroelectric generation by, among others, Koopmans [18] and Bauer et al. [2]. The corresponding questions for pumped storage of energy and other goods have received less attention, and existing models of such technologies lack verisimilitude. ${ }^{1}$ To fill this gap we set up a realistic but tractable model of pumped storage (PS), and we analyse plant operation and valuation in the framework of short-run profit maximisation. Given a time-of-use (TOU) market price $p(t)$ for the good in question (say, electricity), an optimal TOU value $\psi(t)$ is imputed to the stock (of energy converted to a storable form). This solves the operation problem: see (8.2). It therefore makes sense to value the plant's capacities by their marginal contributions to the maximum operating profit, $\Pi_{\mathrm{SR}}^{\mathrm{PS}}$; and these efficiency rents can be expressed in terms of $p$ and $\psi$ (Theorem 9). The rental values can serve as guides to investment (Section 11).

When the given tariff $p$ is a continuous function of time, the stock's shadow price function $\psi$ is unique, and it follows that the capital inputs - viz., the reservoir and the converter or "pump-turbine" - have definite and separate marginal values, $\partial \Pi_{\mathrm{SR}} / \partial k_{\mathrm{St}}$ and $\partial \Pi_{\mathrm{SR}} / \partial k_{\mathrm{Co}}$. Their ratio gives a well-defined rate of substitution in product value terms. This is a striking property because the inputs are also perfect complementsin the sense that no input substitution is possible after fixing the output bundle. That is, the conditional input demands for the storage and conversion capacities depend only on the trajectory of net output from storage, $y(t)$, over the cycle $[0, T]$.

That perfect complements can substitute for each other may seem paradoxical, and of course it would be impossible with a homogeneous, one-dimensional output good: in such a case the output from an input bundle $k$ could only have the familiar fixedcoefficients form $\min \left\{k_{1}, k_{2}, \ldots\right\}$. But with a multi-dimensional, differentiated output good, perfect complementarity would imply fixed input proportions only if the output proportions were fixed - and they are not. With output proportions (as well as scale) allowed to vary, it is the output price system $p$ that aggregates the output bundle $y$ into a scalar, viz., the revenue; and, given a suitable $p$, substitution in revenue terms is possible. With multiple outputs, the inputs can be perfect complements without, like a nut and bolt, having to be used in a fixed proportion.

The problem of maximising the operating profit of a storage plant can be formulated as a linear programme (4.4)-(4.8). Its dual (5.1)-(5.6) is the problem of minimising the plant's value subject to a constraint which decomposes the given price $p$ into a sum of the values of the plant's capital services. The dual can be reformulated as a

\footnotetext{
${ }^{1}$ The existing literature disregards one or both of the main factors in pumped storage, viz., the storage capacity cost and the conversion capacity cost. Pyatt $[21$, p. $752,(10)]$ assumes that there is no capacity constraint on the stock. Nguyen [20, pp. 242-243] excludes both types of capacity cost and concentrates on the running cost (which is of little importance in pumped storage). Gravelle [6] limits his treatment to a two-subperiod model which loses the distinction between the different kinds of storage costs.
} 
problem of shadow pricing for the stock (7.5)-(7.7); this change of variables makes the analysis more transparent and leads to new insights.

The imputed capacity values are useful in planning investment, either as an expansion of existing plants or as a large-scale development of new sites. We formulate the investment problem and show how to solve it for the optimal capacities by equating their marginal values to their marginal costs: see (11.1)-(11.2) and (11.6)-(11.7). It is worth noting that the marginal values are, explicitly or implicitly, essential for any profit-based appraisal of investment plans. Even a comparison of just two alternatives, $k^{\prime}$ and $k^{\prime \prime}$, requires the knowledge of $\Pi_{\mathrm{SR}}^{\mathrm{PS}}(p, k)$ for $k=k^{\prime}, k^{\prime \prime}$, but there is no explicit formula for $\Pi_{\mathrm{SR}}$ (except with the crudest of tariffs, such as the two-valued $p$ of Example 10). By contrast, once the marginals $\nabla_{k} \Pi$ are known, the total profit can be evaluated as $\Pi(k)=\nabla_{k} \Pi \cdot k_{.}{ }^{2}$ The computation of $\Pi$ as the dual value amounts to the same: see Section 9. (And although $\Pi$ could be evaluated as the primal value, the successful algorithms exploit duality and provide the dual solution along with the primal one.)

For its general approach - viz., a continuous-time treatment of storage rents - this study takes inspiration from Koopmans' pioneering paper [18] on optimal water storage policies for a hydro-thermal electricity generating system. In all other respects, however, our work is different. One of our main purposes is to provide a flexible, general framework for dealing with a whole class of problems, whilst Koopmans' analysis is limited to storage hydro - i.e., the storage of a given, natural inflow - and it does not readily extend to similar technologies such as pumped storage. Furthermore, our profit-imputed rental values are unique - unlike Koopmans' rents, which are typically nonunique as a result of being imputed from the saving on the (thermal) operating cost. Also, our dual programme is a simple and direct way of deriving the marginal values, whereas Koopmans' rents are given in terms of a complex operating solution: they do serve his main purpose - which is to verify the cost-optimality of the storage policy he constructs - but the nonuniqueness and complexity of the construction are obstacles to their use in practical investment analysis.

In the SR cost minimisation framework, a production technique with practically no operating cost, such as energy storage, can be studied only in conjunction with others that do have variable costs - such as the thermal fuel cost in Koopmans' problem. By contrast, the profit-maximum formulation allows such a technique to be analysed separately; and this approach is better suited to the more decentralised structure of today's utilities. Also, the switch from cost minimisation to profit maximisation is actually essential for removing the indeterminacy of marginal capacity values. This is because valuations of the storage plant's capacities depend on two time-of-use (TOU) prices, $p(t)$ for the marketed good and $\psi(t)$ for the stock. In the cost minimum approach, both commodities must be shadow-priced; but in competitive SR profit maximisation the good's price function $p$ is treated as given. A possible indeterminacy

\footnotetext{
${ }^{2}$ This identity can also be used to divide the plant's total rent between the fixed inputs on marginalist principles.
} 
of $\psi$ is excluded by a problem-specific argument, viz., an examination of the Lagrange multipliers for the capacity constraints (Lemma 8). ${ }^{3}$

Profit-based valuation of capital inputs is of interest not only to a privately-owned industry, but also to a publicly-owned (or regulated) utility which aims to price its outputs at long-run marginal cost (LRMC) and optimise its capital stock on the basis of short-run calculations. This can be achieved even when - as in peak-load pricing - the SRC is nondifferentiable, but it requires our extension of the WongViner Envelope Theorem [15]. In such a case the familiar LR condition of fixed-input optimality is insufficient. It is replaced with the equality of the inputs' rental prices to their marginal values, but these must be imputed by the SR profit (and not the SR cost).

Continuity over time is the only assumption on $p$ to guarantee a unique solution for $\psi$ (and hence also for the marginal capacity values). Discretisation of time is, however, useful in solving the relevant linear (or convex) programmes by standard numerical methods; and in this context uniqueness of the continuous-time solution ensures that the approximate solutions converge as the discretisation is refined.

Time-continuity of the good's price $p$ is not only a natural assumption to make; it is also one which we verify for competitive equilibrium in [16], where the price function is proved to be continuous for a class of problems that includes peak-load pricing with storage. Our general equilibrium model is set up in a commodity space of bounded functions of time. In part, it is an application of Bewley's framework [3], which we adapt and extend in [10] and [11], as well as providing some mathematical tools in [7], [8] and [14]. It is hoped that this will lead to an integration of hitherto largely separate economic, engineering and OR studies of topics such as peak-load pricing and energy storage.

Section 2 describes the technology. In Sections 4 and 5 the SR profit maximum problem and its dual are set up as linear programmes (LP's) which are doubly infinite: with continuous-time dating of commodities, the primal (4.4)-(4.8) contains a continuum of output variables $y$ and also a continuum of dated capacity constraints (on the flow $y$ and on the stock $s$ ). The primal and the dual are shown to be soluble, and their (optimal) solutions are characterised in Section 6. In Section 7 the dual LP is reformulated as an unconstrained convex programme (CP) for shadow pricing of stock. In Sections 8 and 9 the stock's optimal price $\psi$ is shown to be unique if the good's price $p$ is continuous over time; and formulae are given, in terms of $\psi$, for the optimal output $\hat{y}$ and for $\Pi_{\mathrm{SR}}^{\mathrm{PS}}$ and its derivatives w.r.t. $k_{\mathrm{St}}$ and $k_{\mathrm{Co}}$, which represent the marginal values of the reservoir and the converter. These values are the basis for calculating the optimum investment, in Section 11. The formal presentation (with proofs in the Appendix) is preceded by heuristics, in Section 3. Lastly, Section 12

\footnotetext{
${ }^{3}$ To simplify the exposition we assume here that conversion involves no losses. With imperfect conversion, an optimal $\psi$ is nonunique, but not at the times which matter; so $\nabla_{k} \Pi_{\mathrm{SR}}^{\mathrm{PS}}$ exists even then, as we show in [9].
} 
presents a counterexample to the existence of $\nabla_{k} \Pi$ when the price $p$ is a discontinuous step function (so that time is effectively a discrete variable).

\section{Pumped-Storage technology}

Consider a cyclically priced good that, once put in storage, can be held at no running cost (or loss of stock), as long as the stock does not exceed the reservoir's capacity, $k_{\mathrm{St}}$. The reservoir is charged and discharged with converters; the equipment is so called because the good itself is actually nonstorable (or too costly to store directly), and so it must first be converted into a storable medium. This is a purely intermediate commodity, useful only for reconversion to the original good. Examples include gas liquefaction and conversion of electricity to a storable form of energy: in both cases the running cost of storage is negligible. We refer to each of these techniques as pumped storage (PS). The one closest to our model is the superconducting magnetic energy storage (SMES), in which AC electricity (the good) is converted by a reversible inverter into DC electricity (the medium) to be stored in a superconductive coil. In particular, the conversion is near-perfect: its "round-trip" transformation efficiency is over 95\%: see [19]. In [9] we extend the analysis to imperfect conversion, with a round-trip efficiency $\eta<1$. The extension makes the results applicable to pumped-water energy storage and compressed-air energy storage (PWES and CAES, in which ca. $0.7 \mathrm{kWh}$ is recovered from a $\mathrm{kWh}$ used up).

Here, conversion is assumed to be perfectly efficient, i.e., to involve no losses. Also, the conversion equipment is taken to be symmetrically reversible, i.e., to be capable of transforming the good into the medium and vice versa. Both transformations are taken to be instantaneous (although a constant lag can be readily taken into account). In other words, in unit time a unit of converter can either turn a unit of the good into a unit of the storable medium, or turn a unit of the medium into a unit of the good. A converter's capacity, $k_{\mathrm{Co}}$, is its maximum output rate (in either mode, charging or discharging, because of the symmetry). Note that $k_{\mathrm{St}} / k_{\mathrm{Co}}$ is the time needed to fully charge or discharge the reservoir; and in energy storage $k_{\mathrm{Co}}$ is measured in $\mathrm{kW}$, whereas $k_{\mathrm{St}}$ is measured in $\mathrm{kWh}$.

A PS plant's net output rate (a.k.a. storage policy) is a signed, periodic function $y$, defined on the time interval $[0, T]$ which represents one price cycle. The nonnegative and nonpositive parts, $y^{+}$and $y^{-}$, represent the outflow of the good (from plant to market) and the inflow (from market to plant); these must balance over the cycle, i.e., $\int_{0}^{T} y(t) \mathrm{d} t=0$.

The stock of medium, $s(t)$ at time $t$, is an absolutely continuous function on $[0, T]$ that satisfies the evolution equation $\dot{s}:=\mathrm{d} s / \mathrm{d} t=-y$. This can be restated as

$$
s(t)=s(0)-\int_{0}^{t} y(\tau) \mathrm{d} \tau .
$$

So $s$ is actually a Lipschitz function, since $y$ is essentially bounded ( $k_{\mathrm{Co}} \geq y \geq-k_{\mathrm{Co}}$ a.e.). 
Definition: A real-valued measurable function $y:[0, T] \rightarrow \mathbb{R}$ is essentially bounded, with respect to the Lebesgue measure (meas), if $y$ is bounded on $[0, T] \backslash N$ for some set $N$ with meas $N=0$. Functions which are equal almost everywhere (a.e.) - i.e., differ only on a set of measure zero - are identified with each other. The vector space of all the equivalence classes of essentially bounded functions is denoted by $L^{\infty}[0, T]$. It is normed by the supremum norm

$$
\|y\|_{\infty}:=\operatorname{ess} \sup _{t \in[0, T]}|y(t)|:=\inf _{N: \text { meas } N=0} \sup _{t \in[0, T] \backslash N}|y(t)| .
$$

For the use of $L^{\infty}$ as a commodity space in an equilibrium model of peak-load pricing, see [11] and [16]. For some other uses of $L^{\infty}$, with a general underlying measure (instead of meas), see [3].

The space of all continuous functions $\mathcal{C}[0, T]$, which contains the Lipschitz functions, is normed by the maximum norm

$$
\|s\|_{\infty}=\operatorname{Max}|s|=\max _{t \in[0, T]}|s(t)|
$$

Its norm-dual $\mathcal{C}^{*}$, which serves as the price space for the services of storage capacity, is identified as the space of all Borel measures $\mathcal{M}[0, T]$ by means of the bilinear form

$$
\langle\mu, s\rangle:=\int_{[0, T]} s(t) \mu(\mathrm{d} t)
$$

for $s \in \mathcal{C}$ and $\mu \in \mathcal{M}$ (Riesz's Representation Theorem).

Definition: A (signed, finite) Borel measure on $[0, T]$ is a countably additive realvalued set function on the Borel sigma-algebra; ${ }^{4}$ i.e., it is a $\mu: \mathfrak{B}[0, T] \rightarrow \mathbb{R}$ with $\mu\left(\bigcup_{m=1}^{\infty} B_{m}\right)=\sum_{m=1}^{\infty} \mu\left(B_{m}\right)$ for every sequence of pairwise disjoint sets $B_{m} \in \mathfrak{B}[0, T]$. The vector space of all Borel measures on $[0, T]$ is denoted by $\mathcal{M}[0, T]$. For some of its uses in optimisation, see, e.g., [1] and [17].

The available capacities are taken to equal the installed capacities, and therefore to be constant over the cycle. This does play a part in some of the main results, including the determinacy of rental values (Lemma 8 and Theorem 9). However, to exploit fully the framework of sensitivity analysis, the constant existing capacities $k$ are perturbed with increments $\Delta k$ which are (periodic) functions of time; this is further explained in Section 5. (The notation $\Delta k$, etc., is always to be interpreted as a single symbol meaning "an increment to $k$ ".)

On the assumption of constant capacities $k_{\mathrm{PS}}=\left(k_{\mathrm{St}}, k_{\mathrm{Co}}\right)$, the long-run (LR) production set of the PS technique (with reversible conversion equipment) is the convex

\footnotetext{
${ }^{4}$ The Borel algebra $\mathfrak{B}[0, T]$ is the smallest collection of subsets of $[0, T]$ that contains all the open sets and is closed under set-theoretic operations on a countable sequence of sets.
} 
cone

$$
\begin{aligned}
\mathbb{Y}_{\mathrm{PS}}:= & \left\{\left(y,-k_{\mathrm{PS}}\right) \in L^{\infty} \times \mathbb{R}_{-}^{2}:-k_{\mathrm{Co}} \leq y \leq k_{\mathrm{Co}}, \int_{0}^{T} y(t) \mathrm{d} t=0\right. \\
& \text { and } \left.\exists s_{0} \in \mathbb{R} \forall t \in[0, T] 0 \leq s_{0}-\int_{0}^{t} y(\tau) \mathrm{d} \tau \leq k_{\mathrm{St}}\right\} .
\end{aligned}
$$

This formulation imposes the periodicity or balance constraint $s(T)=s(0)$, but the stock level at the beginning or end of a cycle is taken to be a costless decision variable. In other words, when it is first commissioned, the reservoir comes charged up to any required level at no extra cost, but its periodic operation thereafter is taken to be a technological constraint.

\section{Heuristics for VAluation OF STOCK AND CAPACITIES}

To start with, assume that not only the good's market price, $p(t)$, but also the stored medium's shadow price, $\psi(t)$, is known. Then the operating decisions can be decentralised within the storage plant, with the reservoir "buying" or "selling" the medium at the price $\psi(t)$ to or from the converter, who buys or sells the good at the market price $p(t)$ outside the plant. The SR profit maximisation separates into two problems with obvious solutions, one for each kind of capacity. The maximum profits of the storage and the conversion capacities, $\Pi^{\mathrm{St}}\left(\psi, k_{\mathrm{St}}\right)$ and $\Pi^{\mathrm{Co}}\left(p-\psi, k_{\mathrm{Co}}\right)$, are both linear in $k$. A unit converter can earn the profit flow $(p-\psi)^{-}$by putting the good into storage when $p(t)<\psi(t)$, as well as earning $(p-\psi)^{+}$by taking the good out of storage when $p(t)>\psi(t)$. In both modes, profits are earned only at the times of full capacity utilisation, since the optimum output is $y(t)= \pm k_{\mathrm{Co}}$ whenever $\psi(t) \neq p(t)$ : see Figures $1 \mathrm{a}$ and $1 \mathrm{~b}$. In total over the cycle, the value of a unit converter is therefore $\Pi^{\mathrm{Co}} / k_{\mathrm{Co}}=\int_{0}^{T}|p(t)-\psi(t)| \mathrm{d} t$. As for the reservoir, a unit can earn a profit of $\psi(\bar{\tau})-\psi(\underline{\tau})$ by buying stock at a time $\underline{\tau}$ and selling it at a later time $\bar{\tau}$ when $\psi(\bar{\tau})>\psi(\underline{\tau})$. The value of a unit reservoir is therefore the sum of all shadow price rises in a cycle. In precise terms: if $\psi(T) \geq \psi(0)$, then $\Pi^{\mathrm{St}} / k_{\mathrm{St}}=\operatorname{Var}^{+}(\psi)$, which denotes the total positive variation (a.k.a. upper variation) of $\psi$, i.e., the supremum of $\sum_{m}\left(\psi\left(\bar{\tau}_{m}\right)-\psi\left(\underline{\tau}_{m}\right)\right)^{+}$over all finite sets of pairwise disjoint subintervals $\left(\underline{\tau}_{m}, \bar{\tau}_{m}\right)$ of $(0, T)$. (For a discussion of $\operatorname{Var}^{+}$see, e.g., [5, Section 8.1].) If $\psi(T)<\psi(0)$, the reservoir should start the cycle full, and refill towards the end of the cycle. This brings an extra profit of $\psi(0)-\psi(T)$, so in general the unit rent is the cyclic positive variation

$$
\operatorname{Var}_{\mathrm{c}}^{+}(\psi):=\operatorname{Var}^{+}(\psi)+(\psi(0)-\psi(T))^{+} .
$$

It is later shown that actually $\psi(0)=\psi(T)$ if $p(0)=p(T)$ and $p \in \mathcal{C}[0, T]$.

The maximum operating profit of the whole PS plant $\left(\Pi_{\mathrm{SR}}^{\mathrm{PS}}\right)$ is, however, a function not of $\psi$ but of the problem's parameters $\left(p, k_{\mathrm{PS}}\right)$ alone: $\psi$ is an auxiliary function which must eventually be given in terms of $\left(p, k_{\mathrm{PS}}\right)$. Then $\partial \Pi_{\mathrm{SR}}^{\mathrm{PS}} / \partial k_{\mathrm{St}}$ and $\partial \Pi_{\mathrm{SR}}^{\mathrm{PS}} / \partial k_{\mathrm{Co}}$ 
can be obtained by substituting the correct $\psi$ into the expressions $\operatorname{Var}_{\mathrm{c}}^{+}(\psi)$ and $\int_{0}^{T}|p(t)-\psi(t)| \mathrm{d} t$.

The correct value, $\hat{\psi}$, is that stock price function which minimises the total value of the PS plant's fixed resources $k_{\mathrm{PS}}$. So, given a cyclic TOU tariff $p$, one can find $\hat{\psi}$ by unconstrained minimisation of

$$
k_{\mathrm{St}} \operatorname{Var}_{\mathrm{c}}^{+}(\psi)+k_{\mathrm{Co}} \int_{0}^{T}|p(t)-\psi(t)| \mathrm{d} t
$$

over $\psi$, an arbitrary bounded-variation function on $(0, T)$.

The main feature of this programme is the trade-off between minimising the variation (which on its own would require setting $\psi$ at a constant value) and minimising the integral (which on its own would require setting $\psi$ equal to $p$ ). From this trade-off it is clear to what extent the local peaks of $p$ should be "shaved off" and the troughs "filled in" to obtain the optimum shadow price $\hat{\psi}$ - at least in the case of a piecewise strictly monotone market price $p$. The solution, presented graphically in Figure 1a, is determined by constancy intervals of $\hat{\psi}$, on each of which either $p(t)<\hat{\psi}$ throughout (around a trough of $p$ ) or $p(t)>\hat{\psi}$ throughout (around a local peak of $p$ ). Unless $k_{\mathrm{St}} / k_{\mathrm{Co}}$ is relatively long, these intervals do not abut, and must all be of that length. This is the first-order condition (FOC) for the dual optimum: the increment in the minimand (3.2) that results from shifting the constant value of $\psi$ up or down by an infinitesimal unit, on an interval of length $\tau$, is $\pm\left(k_{\mathrm{St}}-k_{\mathrm{Co}} \tau\right)$. Equating this to zero gives the optimum as $\hat{\tau}=k_{\mathrm{St}} / k_{\mathrm{Co}}$, i.e., $k_{\mathrm{St}} / k_{\mathrm{Co}}$ is the common length of the intervals on which alternately $\hat{\psi}>p$ or $\hat{\psi}<p .{ }^{5}$ This makes it feasible to produce the "bang-coast-bang" output (viz., $y= \pm k_{\text {Co }}$ when $\hat{\psi} \neq p$, with $y=0$ when $\hat{\psi}=p$ ): the reservoir goes alternately from empty to full and vice versa (Figures 1b and 1c). This is the optimal output.

\footnotetext{
${ }^{5}$ Matters complicate when the ratio $k_{\mathrm{St}} / k_{\mathrm{Co}}$ is comparable to the times elapsed between the successive local peaks and troughs of $p$, so that the neighbouring constancy intervals of $\hat{\psi}$ start to abut; but a similar optimality rule applies to such clusters: see [9].
} 


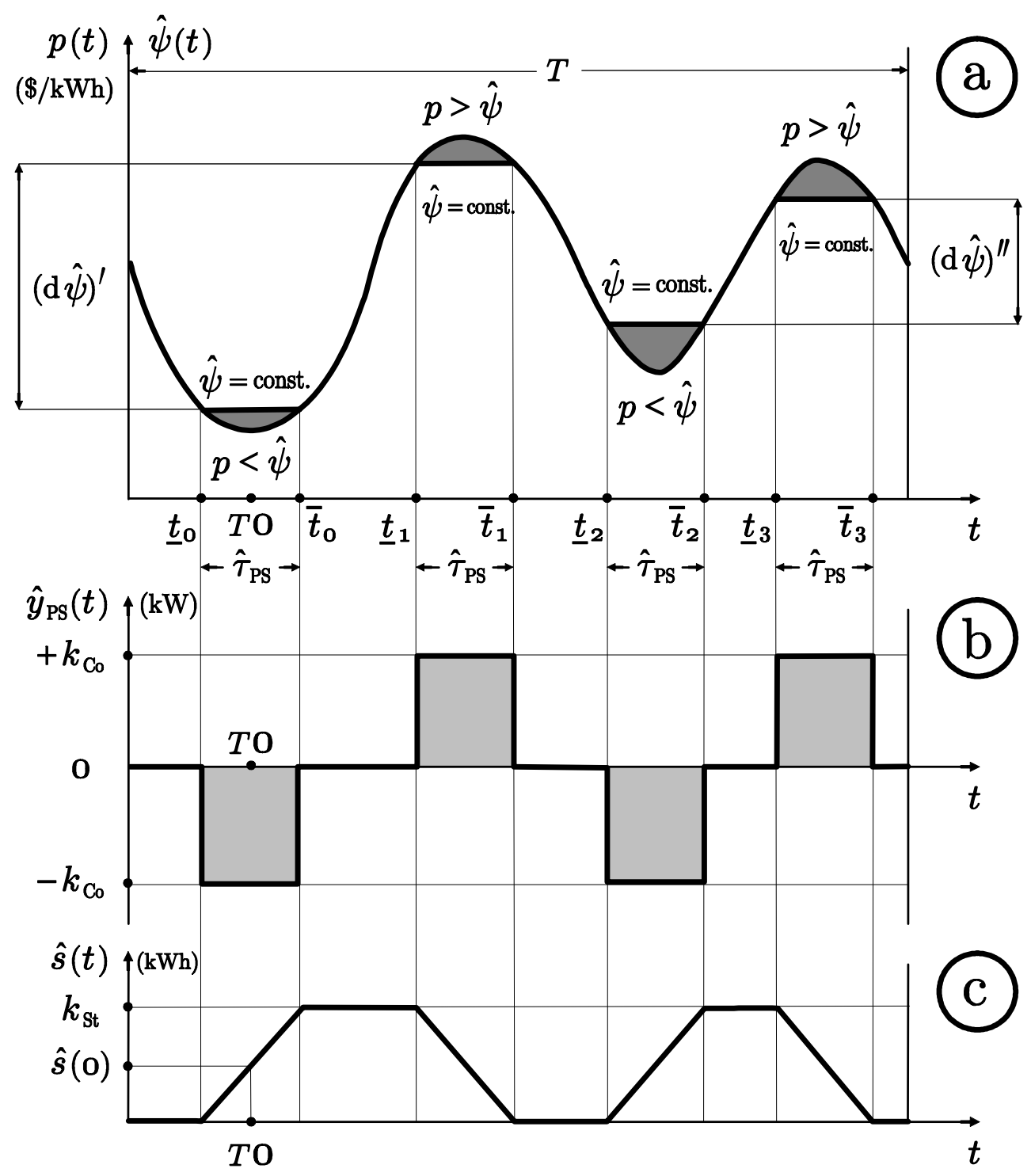

FiguRE 1. Trajectories of: (a) the optimal shadow price $\hat{\psi}$ of stock, (b) the output of pumped-storage plant $\hat{y}_{\mathrm{PS}}$, and (c) the stock $\hat{s}$. Marginal value of storage capacity is $\operatorname{Var}_{\mathrm{c}}^{+}(\hat{\psi})=(\mathrm{d} \hat{\psi})^{\prime}+(\mathrm{d} \hat{\psi})^{\prime \prime}$, the sum of rises of $\hat{\psi}$. Marginal value of conversion capacity is $\int_{0}^{T}|p(t)-\hat{\psi}(t)| \mathrm{d} t$, the sum of dark grey areas in (a). In (b), each of the light grey areas equals the reservoir's capacity $k_{\mathrm{St}}$. By definition $\hat{\tau}_{\mathrm{PS}}=k_{\mathrm{St}} / k_{\mathrm{Co}}$. 


\section{The Linear PRogramme of PLANT OPERATION}

In terms of the production set (2.2), the problem of profit-maximising operation of a PS plant is:

$$
\begin{aligned}
& \text { Given }\left(p, k_{\mathrm{PS}}\right) \in L^{1}[0, T] \times \mathbb{R}_{+}^{2} \\
& \text { maximise } \int_{0}^{T} p(t) y(t) \mathrm{d} t \text { over } y \in L^{\infty}[0, T] \\
& \text { subject to: }\left(y,-k_{\mathrm{PS}}\right) \in \mathbb{Y}_{\mathrm{PS}} \text { defined by }(2.2) \text {. }
\end{aligned}
$$

Notation: The optimal value of (4.1)-(4.3) is the (maximum) operating profit of the PS plant, denoted by $\Pi_{\mathrm{SR}}^{\mathrm{PS}}\left(p, k_{\mathrm{PS}}\right)$. The (optimal) solution set is $\hat{Y}\left(p, k_{\mathrm{PS}}\right)$, occasionally abbreviated to $Y$. The corresponding lowercase notation $\hat{y}$ is used only when the solution is known to be unique. Also, the space $L^{1}$ appearing in (4.1) consists of all functions integrable with respect to (w.r.t.) meas, the Lebesgue measure. The integral $\int_{0}^{T} p(t) y(t) \mathrm{d} t$ is also written as $\langle p, y\rangle$.

The plant operation problem can be formulated as an LP. With the constants $k_{\mathrm{St}}$ and $k_{\mathrm{Co}}$ viewed as special cases of cyclically varying functions, this primal LP is:

(4.4) Given $\left(p ; k_{\mathrm{St}}, k_{\mathrm{Co}}\right) \in L^{1}[0, T] \times \mathbb{R}_{+} \times \mathbb{R}_{+} \subset L^{1}[0, T] \times \mathcal{C}_{+}[0, T] \times L_{+}^{\infty}[0, T]$

$$
\text { maximise } \int_{0}^{T} p(t) y(t) \mathrm{d} t \text { over } y \in L^{\infty}[0, T] \text { and } s_{0} \in \mathbb{R}
$$

(4.6) $\quad$ subject to: $\quad-k_{\mathrm{Co}} \leq y(t) \leq k_{\mathrm{Co}}$ for a.e. $t$

$$
\begin{aligned}
& \int_{0}^{T} y(t) \mathrm{d} t=0 \\
& 0 \leq s_{0}-\int_{0}^{t} y(\tau) \mathrm{d} \tau \leq k_{\mathrm{St}} \quad \text { for every } t .
\end{aligned}
$$

The two formulations of the operation problem are equivalent in the sense that $y$ solves (4.1)-(4.3) if and only if $y$ together with some $s_{0}$ solves (4.4)-(4.8) - in which case $y$ together with the specific value

$$
\underline{s}_{0, y}:=\max _{t \in[0, T]} \int_{0}^{t} y(\tau) \mathrm{d} \tau
$$

is a solution: $\underline{s}_{0, y}$ is the lowest initial stock required for $s(t)$ never to fall below 0 . (Unless there is spare storage capacity, this is actually the only feasible value for $s_{0}$, given $y$.) One can therefore restrict attention to points $\left(y, s_{0}\right)$ with $s_{0}=\underline{s}_{0, y}$; and so the stock trajectory associated with an output $y$ is

$$
s(t)=\underline{s}_{0, y}-\int_{0}^{t} y(\tau) \mathrm{d} \tau .
$$

The dual programme, introduced next, serves the purposes of characterising optimal operation and calculating the marginal capacity values. To ensure that the 
problem is nontrivial - and that the dual is soluble - for the most part it is assumed from here on that $k_{\mathrm{PS}} \gg 0$, i.e., that ${ }^{6}$

$$
k_{\mathrm{Co}}>0 \text { and } k_{\mathrm{St}}>0 .
$$

\section{Capacity valuation as the dual linear programme}

As is set out in, e.g., [22], the dual to a linear or convex programme depends on the choice of perturbations for the primal parameters. A choice of admissible perturbations determines the structure of dual variables (a.k.a. Lagrange multipliers) to be paired with the parameter increments. Therefore the dual programme depends not only on the particular values of primal parameters, but also on the vector space of parameter increments or perturbations. This "ambient" space for the given parameter point can be chosen to suit one's purpose.

In the case of (4.4)-(4.8), the programme contains a separate set of capacity constraints for each time $t$ - and therefore, by considering a separate increment $\Delta k_{\mathrm{PS}}(t)$ for each $t$, one can impute an instantaneous value at each time; i.e., a whole trajectory, $\kappa_{\mathrm{PS}}$, of the values of capital services over the cycle can be determined. Thus the value of capacity services can be separated over time, rather than only determined in total for the cycle. This approach - the introduction of cyclically varying increments $\Delta k_{\mathrm{PS}}$ - is useful even if the existing capacities $k_{\mathrm{PS}}$ are actually taken to be constant. It gives a marginal interpretation to the time-dependent multipliers $\kappa_{\mathrm{PS}}$ (and $\nu_{\mathrm{St}}$ ), which are terms of the TOU price $p$ as per (5.6) below.

As part of this "variation of constants", we consider a cyclically varying increment $\Delta n_{\mathrm{St}}(t)$ to the zero floor for the stock in (4.8). Similarly, a scalar $\Delta \zeta$ is an increment to the zero on the r.h.s. of (4.7); this can be thought of as a quantity of the medium taken to be available for topping up the reservoir between cycles.

Furthermore, we uncouple the converter's charging and discharging modes of operation by considering separate increments, $\Delta k_{\mathrm{Pu}}(t)$ and $\Delta k_{\mathrm{Tu}}(t)$, to its capacity as a "pump" and as a "turbine"; i.e., (4.6) is perturbed to:

$$
-k_{\mathrm{Co}}-\Delta k_{\mathrm{Pu}} \leq y \leq k_{\mathrm{Co}}+\Delta k_{\mathrm{Tu}} \text {. }
$$

The SR profit maximisation problem (4.4)-(4.8) is thus embedded in the family of perturbed programmes obtained by adding an arbitrary cyclically varying increment $\left(\Delta k_{\mathrm{St}}, \Delta n_{\mathrm{St}}, \Delta k_{\mathrm{Pu}}, \Delta k_{\mathrm{Tu}}\right)$ and a scalar $\Delta \zeta$ to the particular parameter point consisting of the constants $\left(k_{\mathrm{St}}, 0, k_{\mathrm{Co}}, k_{\mathrm{Co}}\right)$ and 0 .

The function spaces for the resource increments, already indicated in (4.4), are: $\mathcal{C}[0, T]$ for $\Delta k_{\mathrm{St}}$ and $\Delta n_{\mathrm{St}}$, and $L^{\infty}[0, T]$ for $\Delta k_{\mathrm{Pu}}$ and $\Delta k_{\mathrm{Tu}}$. These spaces are paired with $\mathcal{M}[0, T]$ and $L^{1}[0, T]$ as the shadow price (multiplier) spaces. The pairing of

\footnotetext{
${ }^{6}$ When $k_{\mathrm{Co}}>0$ but $k_{\mathrm{St}}=0$, the primal and dual values are equal (viz., 0 ), but the dual optimum exists only if $p \in \mathrm{BV}$ (in which case the stock price is $\hat{\psi}=p$ ). A positive $k_{\mathrm{PS}}$ means that the primal meets Slater's Condition. This standard constraint qualification for CP's is, in the infinitedimensional case, useful with LP's as well: see also [1, p. 31.].
} 
$L^{\infty}$ with its norm-dual $L^{\infty *}$ (instead of the smaller space $L^{1}$ ) is also needed, but only in proving the dual's solubility: $\kappa_{\mathrm{Pu}}$ and $\kappa_{\mathrm{Tu}}$ are actually in $L^{1}$ (though only because $p \in L^{1}$ instead of $\left.L^{\infty *}\right)$.

The marginal value of the services of a unit storage reservoir on an arbitrary interval $A \subset[0, T]$ is therefore given by a measure $\kappa_{\mathrm{St}}(A)$. Another measure $\nu_{\mathrm{St}}(A)$ gives the incremental profit from lowering the stock floor by a unit, on $A$. The value of the services, on $A$, of a unit converter in the charging mode (i.e., working as a "pump") is the integral of a $\kappa_{\mathrm{Pu}} \in L^{1}$. Similarly the value of its services in the discharging mode (i.e., working as a "turbine") is the integral, over $A$, of a $\kappa_{\mathrm{Tu}} \in L^{1}$. In sum, the converter's rental flow is $\kappa_{\mathrm{Tu}}+\kappa_{\mathrm{Pu}}$.

The marginal value of stock at the beginning (or end) of cycle is a scalar $\lambda$ (paired with $\Delta \zeta$ ).

As is spelt out next, the dual to the operation programme (4.4)-(4.8) consists in minimising the value of the fixed resources by an admissible choice of their shadow prices $\left(\kappa_{\mathrm{St}}, \nu_{\mathrm{St}}, \kappa_{\mathrm{Pu}}, \kappa_{\mathrm{Tu}}, \lambda\right)$, which are paired with the parameter increments $\left(\Delta k_{\mathrm{St}}\right.$, $\left.-\Delta n_{\mathrm{St}}, \Delta k_{\mathrm{Pu}}, \Delta k_{\mathrm{Tu}}, \Delta \zeta\right)$. The main dual constraint is the decomposition (5.6) of the good's price $p$ into a signed sum of: the pump and turbine capacity charges and the shadow price of stock. Later denoted by $\psi$, the stock's price is the sum of: its initial price $\lambda$, the cumulative of reservoir capacity charges $\kappa_{\mathrm{St}}$, and the cumulative of $-\nu_{\mathrm{St}}$.

Theorem 1 (Fixed-input value minimisation as the dual). The dual of the linear programme (4.4)-(4.8), relative to the specified perturbation and the pairing of the parameter spaces $\mathcal{C}$ and $L^{\infty}$ with $\mathcal{M}$ and $L^{1}$ respectively, is:

Given $\left(p ; k_{\mathrm{St}}, k_{\mathrm{Co}}\right)$ as in $(4.4)$

$$
\text { minimise } k_{\mathrm{St}} \int_{[0, T]} \kappa_{\mathrm{St}}(\mathrm{d} t)+k_{\mathrm{Co}} \int_{0}^{T}\left(\kappa_{\mathrm{Tu}}+\kappa_{\mathrm{Pu}}\right)(t) \mathrm{d} t
$$

$$
\text { over } \lambda \in \mathbb{R} \text { and }\left(\kappa_{\mathrm{St}}, \nu_{\mathrm{St}}, \kappa_{\mathrm{Pu}}, \kappa_{\mathrm{Tu}}\right) \in \mathcal{M}[0, T] \times \mathcal{M}[0, T] \times L^{1}[0, T] \times L^{1}[0, T]
$$

subject to: $\quad\left(\kappa_{\mathrm{St}}, \nu_{\mathrm{St}}, \kappa_{\mathrm{Pu}}, \kappa_{\mathrm{Tu}}\right) \geq 0$

$$
\begin{aligned}
& \kappa_{\mathrm{St}}[0, T]=\nu_{\mathrm{St}}[0, T] \\
& p=\lambda+\left(\kappa_{\mathrm{St}}-\nu_{\mathrm{St}}\right)[0, \cdot]+\kappa_{\mathrm{Tu}}-\kappa_{\mathrm{Pu}} .
\end{aligned}
$$


Remark 2. Under (4.11), any solution to (5.1)-(5.6) has the disjointness properties that

$$
\begin{aligned}
\kappa_{\mathrm{Tu}} \wedge \kappa_{\mathrm{Pu}} & =0 \\
\kappa_{\mathrm{St}} \wedge \nu_{\mathrm{St}} & =0 \quad \text { and } \quad \kappa_{\mathrm{St}}\{0, T\} \wedge \nu_{\mathrm{St}}\{0, T\}=0
\end{aligned}
$$

i.e., it is not optimal for the dual variables to overlap and partly cancel each other out.

\section{Conditions for optimal operation and valuation}

The dual programme (5.1)-(5.6) has a solution (in which $\kappa_{\mathrm{Tu}}$ and $\kappa_{\mathrm{Pu}}$ are in $L^{1}$ because $p \in L^{1}$, whilst $\kappa_{\mathrm{St}}$ and $\nu_{\mathrm{St}}$ are in $\left.\mathcal{M}\right)$. The primal and dual optima are characterised by the Kuhn-Tucker Conditions, which for LP's reduce to feasibility and complementary slackness. Spelt out next, these conditions are later used to determine plant operation in terms of the stock price, and to establish that this shadow price is unique.

Proposition 3 (Dual solubility and optimality conditions). Assume (4.11). Then:

1. The fixed-input value minimisation programme (5.1)-(5.6) has an (optimal) solution

$$
\left(\kappa_{\mathrm{St}}, \nu_{\mathrm{St}}, \kappa_{\mathrm{Pu}}, \kappa_{\mathrm{Tu}} ; \lambda\right) \in \mathcal{M}[0, T] \times \mathcal{M}[0, T] \times L^{1}[0, T] \times L^{1}[0, T] \times \mathbb{R} .
$$

The programme's value is finite and equal to the $S R$ profit $\Pi_{\mathrm{SR}}^{\mathrm{PS}}\left(p ; k_{\mathrm{PS}}\right)$, the optimal value of (4.4)-(4.8).

2. Points $\left(y, \underline{s}_{0, y}\right) \in L^{\infty} \times \mathbb{R}$ and $\left(\kappa_{\mathrm{St}}, \nu_{\mathrm{St}}, \kappa_{\mathrm{Pu}}, \kappa_{\mathrm{Tu}} ; \lambda\right)$ are solutions to, respectively, the primal (4.4)-(4.8) and the dual (5.1)-(5.6) if and only if:

(a) $\left(y, \underline{s}_{0, y}\right)$ and $\left(\kappa_{\mathrm{St}}, \nu_{\mathrm{St}}, \kappa_{\mathrm{Pu}}, \kappa_{\mathrm{Tu}} ; \lambda\right)$ are feasible, i.e., satisfy (4.6)-(4.8) and $(5.4)-(5.6)$

(b) The measure $\kappa_{\mathrm{St}}$ is concentrated on $\left\{t \in[0, T]: s(t)=k_{\mathrm{St}}\right\}$, whilst $\nu_{\mathrm{St}}$ is concentrated on $\{t: s(t)=0\}$, where $s$ is given by (4.9)-(4.10).

(c) The function $\kappa_{\mathrm{Tu}}$ vanishes a.e. outside of $\left\{t: y(t)=k_{\mathrm{Co}}\right\}$, whilst $\kappa_{\mathrm{Pu}}$ vanishes outside of $\left\{t: y(t)=-k_{\mathrm{Co}}\right\}$.

The following reformulation of the dual problem extends its pricing interpretation to the valuation of stock.

\section{Shadow PRicing of STOCK AS The DUAL PROBlem}

By the change of variables from $\left(\lambda, \kappa_{\mathrm{St}}(\mathrm{d} t), \nu_{\mathrm{St}}(\mathrm{d} t)\right)$ to

$$
\psi(t)=\lambda+\left(\kappa_{\mathrm{St}}-\nu_{\mathrm{St}}\right)[0, t] \quad \text { for } t \in(0, T),
$$

and by using the dual constraints (5.5)-(5.6) and the disjointness conditions (5.7)(5.8) to eliminate some of the dual variables, the dual problem can be transformed into one of unconstrained minimisation over $\psi$, an arbitrary bounded-variation function on $(0, T)$. 
Notation: The space BV $(0, T)$ consists of all functions $\psi$ of bounded variation on $(0, T)$ with $\psi(t)$ lying between the left and right limits, $\psi(t-)=\lim _{\tau}{ }_{t} \psi(\tau)$ and $\psi(t+)=\lim _{\tau \backslash t} \psi(\tau) .{ }^{7} \mathrm{~A} \psi \in \mathrm{BV}(0, T)$ is extended by continuity to $[0, T]$; i.e., $\psi(0):=\psi(0+)$ and $\psi(T):=\psi(T-)$. The cyclic positive variation of $\psi$ is defined by (3.1).

If finite numbers $\psi(0-)$ and $\psi(T+)$ are additionally specified, then $\psi \in$ $\mathrm{BV}[0-, T+]$; and such a $\psi$ defines a measure on $[0, T]$ by

$$
\mathrm{d} \psi\left[t^{\prime}, t^{\prime \prime}\right]:=\psi\left(t^{\prime \prime}+\right)-\psi\left(t^{\prime}-\right)
$$

for $t^{\prime} \leq t^{\prime \prime}$. The integral of $s$ w.r.t. the measure $(\mathrm{d} \psi)^{+}$is also written as $\int s(\mathrm{~d} \psi)^{+}$. When $\psi(0-)=\psi(T+)$, the usual variation norm of $(\mathrm{d} \psi)^{+}$equals $\operatorname{Var}_{\mathrm{c}}^{+}(\psi)$.

Formulae (7.1) and

$$
\psi(0-)=\psi(T+)=\lambda
$$

define together a one-to-one map of the set of all those $\left(\lambda, \kappa_{\mathrm{St}}, \nu_{\mathrm{St}}\right)$ satisfying (5.4), (5.5) and (5.8) onto the set of all those $\psi \in \mathrm{BV}[0-, T+]$ with $\psi(0-)=\psi(T+)$ lying between $\psi(0+)$ and $\psi(T-)$. The inverse map is given by (7.3) together with

$$
\kappa_{\mathrm{St}}=(\mathrm{d} \psi)^{+} \quad \text { and } \quad \nu_{\mathrm{St}}=(\mathrm{d} \psi)^{-} .
$$

As for the variables $\left(\kappa_{\mathrm{Pu}}, \kappa_{\mathrm{Tu}}\right)$, these can now be eliminated by using (5.6)-(5.7) to express them as

$$
\kappa_{\mathrm{Pu}}=(p-\psi)^{-} \quad \text { and } \quad \kappa_{\mathrm{Tu}}=(p-\psi)^{+} .
$$

Proposition 4 (Stock pricing as the dual). Assume (4.11). Then the fixed-input value minimisation programme (5.1)-(5.6) is equivalent, through the change of variables, to the following convex programme:

$$
\begin{aligned}
& \text { Given }\left(p, k_{\mathrm{PS}}\right) \in L^{1}[0, T] \times \mathbb{R}_{+}^{2} \\
& \text { minimise } k_{\mathrm{St}} \operatorname{Var}_{\mathrm{c}}^{+}(\psi)+k_{\mathrm{Co}} \int_{0}^{T}|p(t)-\psi(t)| \mathrm{d} t \\
& \text { over } \psi \in \mathrm{BV}(0, T) .
\end{aligned}
$$

Notation: The solution set for $(7.5)-(7.7)$ is denoted by $\hat{\Psi}\left(p, k_{\mathrm{PS}}\right) \neq \emptyset$. Again, the corresponding lowercase notation $\hat{\psi}$ is used only when the dual solution is unique.

The function $\psi$ defined by (7.1) can be interpreted as the shadow price of stock at any time $t$. Heuristically, this follows from the marginal interpretations of $\kappa, \nu$ and $\lambda$ (viz., that $\kappa_{\mathrm{St}}$, as the multiplier for the upper reservoir constraint, represents

\footnotetext{
${ }^{7}$ The one-sided limits exist at every $t$ and are equal nearly everywhere (n.e.), i.e., everywhere except for a countable set. Specification of $\psi(t)$ between $\psi(t-)$ and $\psi(t+)$ is unnecessary.
} 
the reservoir capacity value, with a similar interpretation of the multiplier $\nu_{\mathrm{St}}$ for the lower constraint, whilst $\lambda$ is the stock value at the beginning of cycle). ${ }^{8}$

It is this formulation of the dual that leads to the idea of obtaining $\hat{\psi}$ by "levelling off" the local extremes of $p$ in the way described in Section 3. The insight can be developed into a specialised algorithm outlined in [9]. In this approach the dual is tackled first, in the CP form (7.5)-(7.7), with the primal solution found subsequently. (For comparison, the simplex and other methods for LP's find both solutions simultaneously.)

\section{Determination of optimal storage policy}

A storage plant's operation problem is soluble for every $p \in L^{1}[0, T]$.

Proposition 5 (Primal solubility). For every $p \in L^{1}$ and $k_{\mathrm{PS}} \geq 0$, the SR profitmaximising operation programme (4.4)-(4.8) has an (optimal) solution $\left(y, s_{0}\right)$. Equivalently, the problem (4.1)-(4.3) has a solution, i.e., $\hat{Y}\left(p, k_{\mathrm{PS}}\right) \neq \emptyset$.

Once the dual is solved, so that an optimal $\psi$ is known, the operation problem largely reduces to maximisation of instantaneous profits (as Part 2c of Proposition 3 shows). At each $t$ with $p(t) \neq \psi(t)$, the optimum output $y(t)$ is of the "bang-bang control" type. Any remaining part of an optimal $y$ is a "singular control", which arises at a time $t$ when the instantaneous optimum is multi-valued because $\psi(t)$ equals $p(t)$. This part of $y$ can be determined on the assumption (8.1) that $p$ has no plateau: this ensures that $p(t)=\psi(t)$ only when the reservoir is full or empty; and at those times the output rate must be zero. See Figure 1.

Proposition 6 (PS output with plateau-less price). In addition to $p \in L^{1}[0, T]$ and $k_{\mathrm{PS}} \gg 0$, assume that $p$ has no plateau, i.e., that

$$
\forall \mathrm{p} \in \mathbb{R} \quad \text { meas }\{t: p(t)=\mathrm{p}\}=0 .
$$

If $y \in \hat{Y}\left(p, k_{\mathrm{PS}}\right)$ and $\psi \in \hat{\Psi}\left(p, k_{\mathrm{PS}}\right)$, i.e., y solves (4.1)-(4.3) and $\psi$ solves (7.5)-(7.7), then

$$
y(t)=\left\{\begin{array}{ll}
k_{\mathrm{Co}} & \text { if } p(t)>\psi(t) \\
0 & \text { if } p(t)=\psi(t) \\
-k_{\mathrm{Co}} & \text { if } p(t)<\psi(t)
\end{array} .\right.
$$

So (4.1)-(4.3) has a unique solution $\hat{y}\left(p, k_{\mathrm{PS}}\right){ }^{9}$

\footnotetext{
${ }^{8}$ If the shadow-price interpretation of $\psi$ is to be formalised as a rigorous marginal-value result, an exogeneous inflow of the medium $e \in L^{\infty}$ must be introduced as an additional parameter with its own multiplier $\psi$-i.e., (4.8) is perturbed by replacing $y$ with $y-e$, as in [12] and [13]. Then (7.1) becomes a constraint of the dual problem; and $\hat{\psi}$ equals $\nabla_{e} \Pi_{\mathrm{SR}}$, at $e=0$ in the PS context. (If the dual solutions are nonunique, then every $\psi \in \hat{\Psi}$ is a supergradient of $\Pi$ w.r.t. e.)

${ }^{9}$ Since $y$ is fully determined in terms of any optimal $\psi$ (so $y$ is unique even though $\psi$ may be nonunique unless $p \in \mathcal{C}$ ).
} 


\section{Marginal Capacity values in terms of Stock Price}

By definition, $\Pi_{\mathrm{SR}}^{\mathrm{PS}}\left(p, k_{\mathrm{PS}}\right)$ is the value of the primal (operation) problem, $\max _{y}\langle p, y\rangle$. Since the dual and primal values are equal (Proposition 3), a dual (stock-pricing) solution $\psi$ gives $\Pi$ as the total capacity value (the plant's total rent); and it has the advantage of giving the marginal values $\nabla_{k} \Pi$ as well.

Corollary 7 (Dual calculation of SR profit). Assume that $k_{\mathrm{PS}} \gg 0$. Then, for every $\psi \in \hat{\Psi}\left(p, k_{\mathrm{PS}}\right)$,

$$
\Pi_{\mathrm{SR}}^{\mathrm{PS}}\left(p, k_{\mathrm{PS}}\right)=k_{\mathrm{St}} \operatorname{Var}_{\mathrm{c}}^{+}(\psi)+k_{\mathrm{Co}} \int_{0}^{T}|p(t)-\psi(t)| \mathrm{d} t .
$$

Furthermore, this sum is equal to $\int_{0}^{T} \psi y \mathrm{~d} t+\int_{0}^{T}(p-\psi) y \mathrm{~d} t$ term-by-term, for every $y \in \hat{Y}\left(p, k_{\mathrm{PS}}\right) \cdot{ }^{10}$

Since $\Pi$ is positively homogeneous of degree 1 (a.k.a. linearly homogeneous) in $k,{ }^{11}$ Euler's Theorem shows that if $\Pi$ is differentiable in $k,{ }^{12}$ then

$$
\Pi_{\mathrm{SR}}^{\mathrm{PS}}\left(p, k_{\mathrm{PS}}\right)=k_{\mathrm{St}} \frac{\partial \Pi_{\mathrm{SR}}^{\mathrm{PS}}}{\partial k_{\mathrm{St}}}+k_{\mathrm{Co}} \frac{\partial \Pi_{\mathrm{SR}}^{\mathrm{PS}}}{\partial k_{\mathrm{Co}}} .
$$

A comparison with (9.1) suggests that if there is a unique optimal $\psi$, then the partial derivatives of $\Pi$ do exist and equal the coefficients of $k_{\mathrm{St}}$ and $k_{\mathrm{Co}}$ in (9.1); formally this follows from (7.1) and the marginal interpretation of $\kappa_{\mathrm{St}}$ and $\kappa_{\mathrm{Co}}$ (spelt out in the Proof of Theorem 9). And the optimal stock price $\psi$ is indeed unique if $p$, the TOU price of the good, is continuous over time.

Lemma 8 (Stock price uniqueness and continuity). Assume that $p \in \mathcal{C}[0, T]$ and $k_{\mathrm{PS}}=\left(k_{\mathrm{St}}, k_{\mathrm{Co}}\right) \gg 0$. Then the dual (7.5)-(7.7) has a unique (optimal) solution $\hat{\psi}\left(p, k_{\mathrm{PS}}\right)$, which belongs to $\mathcal{C}[0, T]$. If additionally $p(0)=p(T)$, then also $\hat{\psi}(0)=\hat{\psi}(T)$.

Theorem 9 (Efficiency rents of a storage plant). Assume that $p \in \mathcal{C}[0, T]$. Then the operating profit of a pumped-storage plant-i.e., the value of the primal problem (4.1)-(4.3) - is differentiable with respect to the capacities (of the reservoir and the converter $)$, at every $k_{\mathrm{PS}}=\left(k_{\mathrm{St}}, k_{\mathrm{Co}}\right) \gg 0$. The derivatives defining the unit rents are

\footnotetext{
${ }^{10}$ This shows that the capacity values are equal to the capacities' profits- $\langle p-\psi, y\rangle$ for the converter and $\langle\psi, y\rangle$ for the reservoir - when the shadow price $\psi$ is used to decentralise the operation within the plant (as is described in Section 3.2).

${ }^{11}$ That is, $\Pi(p, \alpha k)=\alpha \Pi(p, k)$ for every scalar $\alpha>0$. Note also that $\hat{Y}$ and $\hat{\Psi}$ are positively homogeneous, in $k$, of degrees 1 and 0 respectively; i.e., $\hat{Y}(p, \alpha k)=\alpha \hat{Y}(p, k)$ and $\hat{\Psi}(p, \alpha k)=\hat{\Psi}(p, k)$ for $\alpha>0$.

${ }^{12}$ If $\Pi$ is nondifferentiable, then $\Pi(k)=r \cdot k$ for every $r \in \partial_{k} \Pi$ (the superdifferential of $\Pi$ as a concave function of $k$ ).
} 
given by the formulae

$$
\begin{aligned}
& \frac{\partial \Pi_{\mathrm{SR}}^{\mathrm{PS}}}{\partial k_{\mathrm{St}}}=\operatorname{Var}_{\mathrm{c}}^{+}\left(\hat{\psi}\left(p, k_{\mathrm{PS}}\right)\right) \\
& \frac{\partial \Pi_{\mathrm{SR}}^{\mathrm{PS}}}{\partial k_{\mathrm{Co}}}=\int_{0}^{T}\left|p(t)-\hat{\psi}\left(p, k_{\mathrm{PS}}\right)(t)\right| \mathrm{d} t,
\end{aligned}
$$

in which $\hat{\psi}$ is the unique solution to the dual problem (7.5)-(7.7) of shadow stock pricing.

\section{BOUNDS ON MARGINAL CAPACITY VALUES}

Since $\Pi_{\mathrm{SR}}^{\mathrm{PS}}$ is the minimum of (7.6) over $\psi \in \mathrm{BV}$, an upper bound on $\Pi / k_{\mathrm{Co}}$ that depends only on $p$ can be obtained by setting $\psi=$ const. in (7.6); and the best constant is the median of $p$ (defined by (A.19)). This and (9.2) show that, when $p \in \mathcal{C}$

$$
\frac{\partial \Pi_{\mathrm{SR}}^{\mathrm{PS}}}{\partial k_{\mathrm{Co}}} \leq \frac{\Pi_{\mathrm{SR}}^{\mathrm{PS}}}{k_{\mathrm{Co}}} \leq \int_{0}^{T}|p(t)-\operatorname{med}(p)| \mathrm{d} t .
$$

When additionally $p \in \mathrm{BV}$, setting $\psi=p$ in (7.6) shows similarly that

$$
\frac{\partial \Pi_{\mathrm{SR}}^{\mathrm{PS}}}{\partial k_{\mathrm{St}}} \leq \frac{\Pi_{\mathrm{SR}}^{\mathrm{PS}}}{k_{\mathrm{St}}} \leq \operatorname{Var}_{\mathrm{c}}^{+}(p) .
$$

With $p$ fixed, $\nabla_{k} \Pi$ is homogeneous of degree 0 in $k$, i.e., it depends only on the capacity ratio $\vartheta:=k_{\mathrm{Co}} / k_{\mathrm{St}}$. As $\vartheta$ increases from 0 to $+\infty, \partial \Pi / \partial k_{\mathrm{St}}$ increases, whilst $\partial \Pi / \partial k_{\mathrm{Co}}$ decreases to 0 (in the limit as $\vartheta \nearrow+\infty$ ), since

$$
\frac{k_{\mathrm{Co}}}{k_{\mathrm{St}}} \frac{\partial \Pi_{\mathrm{SR}}^{\mathrm{PS}}}{\partial k_{\mathrm{Co}}} \leq \frac{\Pi_{\mathrm{SR}}^{\mathrm{PS}}}{k_{\mathrm{St}}} \leq \operatorname{Var}_{\mathrm{c}}^{+}(p)
$$

by (9.2) and the second inequality of (10.2). A similar argument using (10.1) shows that

$$
\frac{k_{\mathrm{St}}}{k_{\mathrm{Co}}} \frac{\partial \Pi_{\mathrm{SR}}^{\mathrm{PS}}}{\partial k_{\mathrm{St}}} \leq \frac{\Pi_{\mathrm{SR}}^{\mathrm{PS}}}{k_{\mathrm{Co}}} \leq \int_{0}^{T}|p(t)-\operatorname{med}(p)| \mathrm{d} t
$$

so $\partial \Pi / \partial k_{\mathrm{St}}$ decreases to 0 as $\vartheta \searrow 0$. However, whilst $\partial \Pi / \partial k_{\mathrm{Co}}$ may never equal $0,{ }^{13} \partial \Pi / \partial k_{\mathrm{St}}$ actually is 0 for small enough $\vartheta=k_{\mathrm{Co}} / k_{\mathrm{St}}$. This is obviously the case for $\vartheta<2 / T$ : an extra unit of the reservoir is then useless (because it is already so large that it cannot be fully charged and discharged in one cycle). The largest $\vartheta$ with $\partial \Pi / \partial k_{\mathrm{St}}=0$ - denoted by $\underline{\vartheta}$ - can be given explicitly in terms of $p .{ }^{14}$ Note that $\partial \Pi / \partial k_{\mathrm{Co}}$ attains its upper bound at (and only at) $\vartheta \leq \underline{\vartheta} .{ }^{15}$ See Figure 2a.

\footnotetext{
${ }^{13}$ If the local peaks and troughs of $p$ are strict, then an extra unit of converter is always useful because it allows conversion to be concentrated closer to the troughs and peaks.

${ }^{14}$ This is done by working out the storage capacity required for the output $k_{\mathrm{Co}} \operatorname{sgn}(p-\operatorname{med}(p))$, under (8.1).

${ }^{15}$ If $\vartheta \leq \underline{\vartheta}$, then $\partial \Pi / \partial k_{\mathrm{St}}=0$; i.e., $\hat{\psi}=\operatorname{med}(p)$, so equalities hold in (10.1).
} 


\section{Optimum investment in Storage Plants}

The marginal capacity values $\nabla_{k} \Pi_{\mathrm{SR}}^{\mathrm{PS}}$ can be used to determine the optimum investment into pumped storage on the basis of a given TOU tariff $p$ and the supply costs of the two inputs, the reservoir and the converter. The following formulation of the problem applies chiefly to energy storage techniques such as PWES and CAES, which utilise special geological features. The converter's unit cost, $r_{\mathrm{Co}}$, can be reasonably regarded as constant, i.e., independent of the capacity $k_{\mathrm{Co}}$. By contrast, in PWES or CAES the reservoir's marginal cost, $r_{\mathrm{St}}$, typically increases with $k_{\mathrm{St}}$ because the most suitable parts of the site are developed first. In formal terms, on a potential site for a particular storage technique, a reservoir can be built at a cost which is a strictly convex and increasing function, $G$, of its capacity $k_{\mathrm{St}} \in\left[0, \bar{k}_{\mathrm{St}}\right]$, with $G(0)=0$. Although $G$ may be nondifferentiable, it has the one-sided, left and right derivatives, $\mathrm{d} G / \mathrm{d}_{-} k_{\mathrm{St}} \leq \mathrm{d} G / \mathrm{d}_{+} k_{\mathrm{St}}$. Where these differ, the subdifferential $\partial G=\left[\mathrm{d} G / \mathrm{d}_{-} k_{\mathrm{St}}, \mathrm{d} G / \mathrm{d}_{+} k_{\mathrm{St}}\right]$ is multi-valued; but this can be the case only on a countable subset of $\left(0, \bar{k}_{\mathrm{St}}\right)$. In other words, the two-sided derivative $\mathrm{d} G / \mathrm{d} k_{\mathrm{St}}$ exists nearly everywhere; and its right or left limit equals $\mathrm{d} G / \mathrm{d}_{ \pm} k_{\mathrm{St}}$, respectively. Also, $\partial G(0)=\left[0,\left(\mathrm{~d} G / \mathrm{d} k_{\mathrm{St}}\right)(0+)\right]$ and $\partial G\left(\bar{k}_{\mathrm{St}}\right)=\left[\left(\mathrm{d} G / \mathrm{d} k_{\mathrm{St}}\right)\left(\bar{k}_{\mathrm{St}}-\right),+\infty\right)$. See Figure $2 \mathrm{~b}$.

The investment problem is:

$$
\begin{aligned}
& \text { Given }\left(p, r_{\mathrm{Co}}\right) \in \mathcal{C}[0, T] \times \mathbb{R}_{++}(\text {and the function } G) \\
& \text { maximise } \Pi_{\mathrm{SR}}^{\mathrm{PS}}\left(p ; k_{\mathrm{St}}, k_{\mathrm{Co}}\right)-G\left(k_{\mathrm{St}}\right)-r_{\mathrm{Co}} k_{\mathrm{Co}} \text { over }\left(k_{\mathrm{St}}, k_{\mathrm{Co}}\right) \in \mathbb{R}_{+}^{2} .
\end{aligned}
$$

It can be solved in two stages, first for the proportion $\vartheta:=k_{\mathrm{Co}} / k_{\mathrm{St}}$, and then for the scale: since $\Pi$ is positively linearly homogeneous in $k$,

$$
\Pi_{\mathrm{SR}}^{\mathrm{PS}}\left(p ; k_{\mathrm{St}}, k_{\mathrm{Co}}\right)-G\left(k_{\mathrm{St}}\right)-r_{\mathrm{Co}} k_{\mathrm{Co}}=k_{\mathrm{St}} \cdot\left(\Pi_{\mathrm{SR}}^{\mathrm{PS}}\left(p ; 1, \frac{k_{\mathrm{Co}}}{k_{\mathrm{St}}}\right)-\frac{r_{\mathrm{Co}} k_{\mathrm{Co}}}{k_{\mathrm{St}}}\right)-G\left(k_{\mathrm{St}}\right)
$$

for $k_{\mathrm{St}}>0$; and - with $p$ suppressed from the notation - the subproblem of maximising

$$
\Pi_{\mathrm{SR}}^{\mathrm{PS}}(1, \vartheta)-r_{\mathrm{Co}} \vartheta
$$

over $\vartheta \in \mathbb{R}_{+}$can be solved first. Once a maximum point $\vartheta$ is known, it only remains to maximise

$$
k_{\mathrm{St}} \cdot\left(\Pi_{\mathrm{SR}}^{\mathrm{PS}}(1, \vartheta)-r_{\mathrm{Co}} \vartheta\right)-G\left(k_{\mathrm{St}}\right)
$$

over $k_{\mathrm{St}} \in \mathbb{R}_{+}$. The solution gives the other optimum capacity as $k_{\mathrm{Co}}=\vartheta k_{\mathrm{St}}$.

When $\max _{\vartheta}\left(\Pi(1, \vartheta)-r_{\mathrm{Co}} \vartheta\right) \leq\left(\mathrm{d} G / \mathrm{d} k_{\mathrm{St}}\right)(0+)$, the maximum of $(11.5)$ is at $k_{\mathrm{St}}=$ 0 , and this means that the maximum of $(11.2)$ is at $\left(k_{\mathrm{St}}, k_{\mathrm{Co}}\right)=(0,0)$. Therefore a necessary condition for a nonzero solution to (11.2) is that $r_{\mathrm{Co}}<\bar{r}_{\mathrm{Co}}:=$ $\int_{0}^{T}|p(t)-\operatorname{med}(p)| \mathrm{d} t$ (because, from Section $10, \bar{r}_{\mathrm{Co}}$ is the maximum of $\partial \Pi / \partial k_{\mathrm{Co}}$, so $\max _{\vartheta}\left(\Pi(1, \vartheta)-r_{\mathrm{Co}} \vartheta\right)=0$ if $\left.r_{\mathrm{Co}} \geq \bar{r}_{\mathrm{Co}}\right)$. 
Given any $r_{\mathrm{Co}}<\bar{r}_{\mathrm{Co}}$, a $\vartheta$ maximising (11.4) can be found from the $\mathrm{FOC}^{16}$

$$
\left.\frac{\partial \Pi_{\mathrm{SR}}^{\mathrm{PS}}}{\partial k_{\mathrm{Co}}}\right|_{\left(k_{\mathrm{St}}, k_{\mathrm{Co}}\right)=(1, \vartheta)}=r_{\mathrm{Co}} .
$$

This has a solution because $\partial \Pi / \partial k_{\mathrm{Co}} \searrow 0$ as $\vartheta \nearrow+\infty$, at least if $p \in \mathrm{BV}$ : see Section 10. In general the maximum points of (11.4) form a (nonempty) subinterval of $(\underline{\vartheta},+\infty)$, but if $p$ has no plateau, then the solution is actually unique, in which case it is denoted by $\vartheta^{*}\left(r_{\mathrm{Co}}\right)$, as in Figure $2 \mathrm{a}$.

Given an optimum $\vartheta$, the $k_{\mathrm{St}}$ maximising (11.5) can be found from the condition $\Pi(1, \vartheta)-r_{\mathrm{Co}} \vartheta \in \partial G\left(k_{\mathrm{St}}\right)$, which is equivalent to

$$
\left.\frac{\partial \Pi_{\mathrm{SR}}^{\mathrm{PS}}}{\partial k_{\mathrm{St}}}\right|_{\left(k_{\mathrm{St}}, k_{\mathrm{Co}}\right)=(1, \vartheta)} \in \partial G\left(k_{\mathrm{St}}\right)
$$

by (11.6) and (9.2). Since $G$ is strictly convex, the solution for $k_{\mathrm{St}}$ is unique: see Figure 2b.

In summary, given an $r_{\mathrm{Co}}<\bar{r}_{\mathrm{Co}}$ and a plateau-less continuous $p$ of bounded variation, there is a unique optimum investment, $k_{\mathrm{St}}^{*}\left(G, r_{\mathrm{Co}}\right)$ and $k_{\mathrm{Co}}^{*}\left(G, r_{\mathrm{Co}}\right)$, which can be found by using $\nabla_{k} \Pi$ : first (11.6) is solved to obtain $\vartheta^{*}\left(r_{\mathrm{Co}}\right)$, and then (11.7) with $\vartheta=\vartheta^{*}$ is solved to obtain $k_{\mathrm{St}}^{*}$ and hence also $k_{\mathrm{Co}}^{*}=\vartheta^{*} k_{\mathrm{St}}^{*}{ }^{17}$

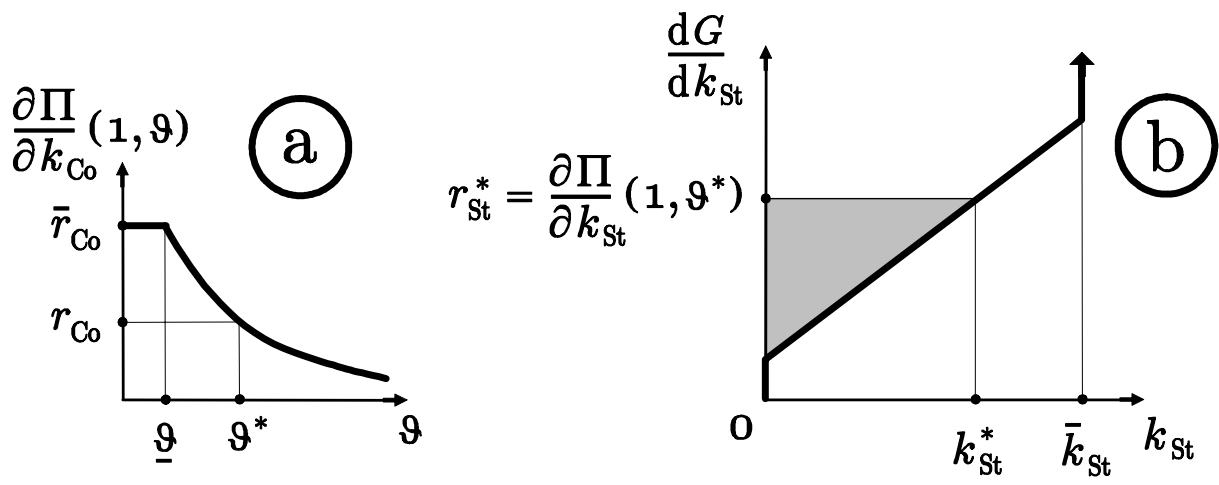

Figure 2. Optimal investment on a storage site: determination of (a) the capacity ratio $\vartheta^{*}$ (given $r_{\mathrm{Co}}$ ), and (b) the reservoir's capacity $k_{\mathrm{St}}^{*}$ (and hence the converter's capacity $k_{\mathrm{Co}}^{*}=\vartheta^{*} k_{\mathrm{St}}^{*}$ ). The shaded area in (b) represents the site's rent.

\footnotetext{
${ }^{16}$ This can be solved numerically by, e.g., the secant method-which requires no more than the calculation of $\partial \Pi / \partial k_{\text {Co }}$ at the successive approximations.

${ }^{17}$ The procedure is valid also when $k_{\mathrm{St}}^{*}=0: \vartheta^{*}$ is the candidate for the optimum capacity ratio, and it can be found without presupposing that the ratio is well defined (i.e., that $k_{\mathrm{St}}^{*}>0$ ).
} 


\section{Comments:}

1. The maximum of (11.2) equals $r_{\mathrm{St}}^{*} k_{\mathrm{St}}^{*}-G\left(k_{\mathrm{St}}^{*}\right)$, where

$$
r_{\mathrm{St}}^{*}\left(r_{\mathrm{Co}}\right):=\frac{\partial \Pi_{\mathrm{SR}}^{\mathrm{PS}}}{\partial k_{\mathrm{St}}}\left(1, \vartheta^{*}\left(r_{\mathrm{Co}}\right)\right)=\frac{\partial \prod_{\mathrm{SR}}^{\mathrm{PS}}}{\partial k_{\mathrm{St}}}\left(k_{\mathrm{St}}^{*}\left(G, r_{\mathrm{Co}}\right), k_{\mathrm{Co}}^{*}\left(G, r_{\mathrm{Co}}\right)\right) .
$$

Since $r_{\mathrm{St}}^{*} \in \partial G\left(k_{\mathrm{St}}^{*}\right)$, this is the price for storage capacity that would induce a price-taking owner of the site to build a reservoir of the optimum size $k_{\mathrm{St}}^{*}$, to be optimally complemented by $k_{\mathrm{Co}}^{*}$ of the converter. In practice the site owner is likely to either build a complete plant himself or let the site for a rent to the highest-bidding entrepreneur. With perfect competition the entrepreneur's net profit is zero, i.e., the rent for the site is $r_{\mathrm{St}}^{*} k_{\mathrm{St}}^{*}-G\left(k_{\mathrm{St}}^{*}\right)$ per cycle (the shaded area in Figure 2b).

2. The analysis obviously extends to any number of sites and techniques (for storing the same good with the tariff $p$ ). On all of the sites for a particular storage technique the optimum capacity ratio $\vartheta^{*}$ is the same, since it depends only on $r_{\mathrm{Co}}$.

3. The independence of $\vartheta^{*}$ on $G$ gives a simple but useful comparative statics result: a fall in the marginal cost schedule to some $\mathrm{d} G^{\prime} / \mathrm{d} k_{\mathrm{St}} \leq \mathrm{d} G / \mathrm{d} k_{\mathrm{St}}$ changes the scale of optimum investment but not the optimal capacity ratio. So if the reservoir construction cost falls to $G^{\prime}$ after an investment on the basis of $G$, optimality can be restored by a proportional expansion of the existing plant. (This is usually feasible with sizeable projects, which are planned to be carried out in stages.)

\section{INDETERMinACy OF MARGinAL VALUeS With DisCRETE TIME}

As is next shown by means of a two-period model, discretisation of time can make $\Pi_{\mathrm{SR}}^{\mathrm{PS}}(p, k)$ nondifferentiable in $k$. This is because it forces $p$ to be piecewise constant and thus discontinuous; and the optimal $\psi$ 's are nonunique if $p$ has a jump paired with a drop at two instants which differ exactly by $k_{\mathrm{St}} / k_{\mathrm{Co}}$ - which is always the case in the two-period model (unless there is spare capacity of one kind or the other). In the following example the cycle is divided into subperiods of lengths $d$ and $T-d$. Then $\Pi(k)$ is proportional to $\min \left\{k_{\mathrm{St}}, \delta k_{\mathrm{Co}}\right\}$, where $\delta:=\min \{d, T-d\}$. The only efficient capacity ratio is $k_{\mathrm{St}} / k_{\mathrm{Co}}=\delta$; and $\Pi$ is obviously nondifferentiable at such a $k$.

The form which $\Pi(k)$ has in the two-period model may create the false impression that storage is a fixed-coefficients technology - but actually even the two-period framework (with a varying $d$ ) reveals that this is not so: although, given a two-valued tariff $p$, there is just one efficient capacity ratio $\delta$, it is not determined by the technology alone because it depends on the price duration $d$ (while being independent of the two price levels in $p$ ). This is why the example is not limited to the case of $d=T / 2$. 
Example 10 (Indeterminacy of marginal values with a discontinuous price). The short-run profit function of the pumped storage technique (2.2) can be nondifferentiable in $k_{\mathrm{PS}}$. To see this, take any numbers $\overline{\mathrm{p}}>\underline{\mathrm{p}} \geq 0$ and $d \in(0, T)$, and set $a$ piecewise constant tariff

$$
p(t):=\left\{\begin{array}{ll}
\underline{\mathrm{p}} & \text { if } t<d \\
\overline{\mathrm{p}} & \text { if } t \geq d
\end{array} .\right.
$$

Then, for a PS plant with capacities $k_{\mathrm{PS}}$, a profit-maximising output is ${ }^{18}$

$$
y(t)= \begin{cases}-\left(k_{\mathrm{St}} \wedge \delta k_{\mathrm{Co}}\right) / d & \text { if } t<d \\ \left(k_{\mathrm{St}} \wedge \delta k_{\mathrm{Co}}\right) /(T-d) & \text { if } t \geq d\end{cases}
$$

where $\delta:=d \wedge(T-d)$. So

$$
\frac{1}{\overline{\mathrm{p}}-\underline{\mathrm{p}}} \Pi_{\mathrm{SR}}^{\mathrm{PS}}\left(p, k_{\mathrm{PS}}\right)=k_{\mathrm{St}} \wedge \delta k_{\mathrm{Co}}:=\min \left\{k_{\mathrm{St}}, \delta k_{\mathrm{Co}}\right\} .
$$

Therefore $\Pi_{\mathrm{SR}}^{\mathrm{PS}}$ is nondifferentiable in $k_{\mathrm{PS}}$ whenever $k_{\mathrm{St}}=\delta k_{\mathrm{Co}}$.

Comments:

1. When $k_{\mathrm{St}}=\delta k_{\mathrm{Co}}$, the superdifferential (the set of supergradients) of $\Pi$, as a concave function of $k_{\mathrm{PS}}$, can be calculated directly from (12.1) as

$$
\partial_{k_{\mathrm{PS}}} \Pi_{\mathrm{SR}}^{\mathrm{PS}}=\left\{r^{\mathrm{PS}}=\left(r^{\mathrm{St}}, r^{\mathrm{Co}}\right) \geq 0: r^{\mathrm{St}}+\frac{1}{\delta} r^{\mathrm{Co}}=\overline{\mathrm{p}}-\underline{\mathrm{p}}\right\} .
$$

2. Each supergradient in $\partial_{k} \Pi$ can also be obtained from an optimal shadow price of stock $\psi \in \hat{\Psi}\left(p, k_{\mathrm{PS}}\right)$. With $\left(p, k_{\mathrm{PS}}\right)$ as above, an optimum $\psi$ is nonunique; and it is almost completely indeterminate if $d=T / 2$ : in this case it is any two-valued function

$$
\psi(t)= \begin{cases}\underline{\psi} & \text { if } t<T / 2 \\ \bar{\psi} & \text { if } t \geq T / 2\end{cases}
$$

subject only to the obvious conditions, viz., that ${ }^{19}$

$$
\underline{\mathrm{p}} \leq \underline{\psi} \leq \bar{\psi} \leq \overline{\mathrm{p}}
$$

\footnotetext{
${ }^{18}$ This $y$ implements the policy of carrying over, from the low-price period to the high-price period, as much stock as the capacity constraints allow, viz., $\min \left\{k_{\mathrm{St}}, \delta k_{\mathrm{Co}}\right\}$. It is optimal independently of the two price levels, as long as $\overline{\mathrm{p}}>\underline{\mathrm{p}}$. (Also, it is the only two-valued optimal output function; but in the class of all functions it is the unique optimum if and only if $d=T / 2$ and $k_{\mathrm{St}} \geq k_{\mathrm{Co}} T / 2$.)

${ }^{19}$ If $d \neq T / 2$ then $d$ replaces $T / 2$ in (12.3), and $\delta$ replaces $T / 2$ in (12.5); but additionally $\bar{\psi}=\overline{\mathrm{p}}$ if $d<T / 2$, and similarly $\underline{\mathrm{p}}=\underline{\psi}$ if $d>T / 2$.
} 
Every $\psi$ satisfying (12.3)-(12.4) gives an $r^{\mathrm{PS}} \in \partial_{k_{\mathrm{PS}}} \Pi$ by the formulae

$$
r^{\mathrm{St}}=\bar{\psi}-\underline{\psi} \quad \text { and } \quad r^{\mathrm{Co}}=\frac{T}{2} \cdot(\underline{\psi}-\underline{\mathrm{p}}+\overline{\mathrm{p}}-\bar{\psi}) .
$$

This is a special case of the derivative property of $\Pi$ stated in (A.21).

\section{Conclusions}

This analysis gives, we believe for the first time, a sound basis for valuation and optimal operation of existing pumped-storage plants, as well as for investment decisions. Our model of the technology distinguishes the different types of capacity within a storage plant, viz., the reservoir and the converter. Their marginal contributions to the operating profit turn out to be well defined, at least when the given TOU price is continuous over the cycle. These values can be calculated by solving a linear programme (or an equivalent convex programme). We have also shown how to use the marginal values to determine the optimum investment into storage. The framework is flexible and can deal with similar storage problems: for example, in [12] and [13] we study hydroelectric generation (which, unlike pumped storage, is a case of storing an exogenous inflow).

\section{Appendix A. Proofs}

Except for the shadow-price uniqueness result (Lemma 8), the proofs are mostly routine applications of duality for optimisation in infinite-dimensional spaces, as expounded in, e.g., [22, Examples 4, 4', 4"] and [1, 3.3-3.7]. To put the primal constraints in the operator form required by this framework, define the integrals $I_{0}$ and $I_{T}: L^{\infty}[0, T] \rightarrow \mathcal{C}[0, T]$ by

$$
\left(I_{0} y\right)(t):=\int_{0}^{t} y(\tau) \mathrm{d} \tau, \quad\left(I_{T} y\right)(t):=\int_{t}^{T} y(\tau) \mathrm{d} \tau .
$$

The reservoir constraints $(4.8)$ on $\left(y, s_{0}\right)$ can then be rewritten as

$$
0 \leq s_{0} 1_{[0, T]}-I_{0} y \leq k_{\text {St }} .
$$

A formula for the adjoint operation $I_{0}^{*}: \mathcal{M}[0, T] \rightarrow L^{\infty *}[0, T]$ is needed. (As for the embedding $\mathbb{R} \ni s_{0} \mapsto s_{0} 1_{[0, T]} \in \mathcal{C}$, its adjoint is: $\mathcal{M} \ni \kappa \mapsto\langle\kappa, 1\rangle=\kappa[0, T]$.)

Lemma 11. The adjoints $I_{0}^{*}, I_{T}^{*}$ map $\mathcal{M}[0, T]$ into $\mathrm{BV}[0, T] \subset L^{1}[0, T]$. They are given by

$$
\left(I_{0}^{*} \mu\right)(t)=\mu[t, T] \quad \text { and } \quad\left(I_{T}^{*} \mu\right)(t)=\mu[0, t] \quad \text { for a.e. } t,
$$

for every $\mu \in \mathcal{M}$. If $\mu[0, T]=0$, then $-I_{0}^{*} \mu=\mu[0, \cdot]=I_{T}^{*} \mu$.

Proof. This follows from Fubini's Theorem: for details, see [9]. 
Proof of Theorem 1 (Fixed-input value minimisation as the dual). Since (4.4)-(4.8) is an LP, it would suffice to apply results such as those of [1, 3.3 and 3.6-3.7]. However, to facilitate extensions and adaptations requiring convex but nonlinear models, this proof is couched in CP terms. The dual to a concave maximisation programme consists in minimising, over the dual variables (the Lagrange multipliers for the primal), the supremum of the Lagrange function over the primal decision variables: see, e.g., $[22,(4.6)$ and (5.13)]. The "cone model" of [22, Example 4'] is applicable, since (A.2) and (4.6) represent the inequality constraints of the primal programme (4.4)-(4.8) by means of the nonnegative cones $\left(\mathcal{C}_{+}\right.$and $\left.L_{+}^{\infty}\right)$ and convex constraint maps (which are actually linear). The dual variables here are the $\kappa_{\mathrm{St}}, \nu_{\mathrm{St}}, \kappa_{\mathrm{Pu}}, \kappa_{\mathrm{Tu}}$ and $\lambda$ of (5.3); and these are paired with the parameter increments $\Delta k_{\mathrm{St}},-\Delta n_{\mathrm{St}}, \Delta k_{\mathrm{Tu}}, \Delta k_{\mathrm{Pu}}$ and $\Delta \zeta$ (as is discussed in Section 5). The primal variables are $\left(y, s_{0}\right) \in L^{\infty} \times \mathbb{R}$, and the Lagrange function (of primal and dual variables) is

$$
\mathcal{L}^{\mathrm{PS}}\left(y, s_{0} ; \kappa, \nu, \lambda\right)= \begin{cases}\Pi_{\mathrm{Exc}}^{\mathrm{PS}}\left(y, s_{0} ; \kappa, \nu, \lambda\right)+V^{\mathrm{PS}}(\kappa) & \text { if }(\kappa, \nu) \geq 0 \\ +\infty & \text { if }(\kappa, \nu) \ngtr 0\end{cases}
$$

where

$$
V^{\mathrm{PS}}:=\left\langle\kappa_{\mathrm{St}}, k_{\mathrm{St}}\right\rangle_{\mathcal{M}, \mathcal{C}}+\left\langle\kappa_{\mathrm{Tu}}+\kappa_{\mathrm{Pu}}, k_{\mathrm{Co}}\right\rangle_{L^{\infty *}, L^{\infty}}
$$

and, with the notation

$$
\mu_{\mathrm{St}}:=\kappa_{\mathrm{St}}-\nu_{\mathrm{St}} \quad \text { and } \quad \mu_{\mathrm{Co}}:=\kappa_{\mathrm{Tu}}-\kappa_{\mathrm{Pu}} .
$$

one has

$$
\begin{aligned}
\Pi_{\mathrm{Exc}}^{\mathrm{PS}} & :=\langle p, y\rangle-\left\langle\kappa_{\mathrm{St}}-\nu_{\mathrm{St}}, s_{0}-I_{0} y\right\rangle-\left\langle\kappa_{\mathrm{Tu}}-\kappa_{\mathrm{Pu}}, y\right\rangle-\lambda\langle 1, y\rangle \\
& =\langle p, y\rangle+\left\langle I_{0}^{*} \mu_{\mathrm{St}}, y\right\rangle-\left\langle\mu_{\mathrm{St}}, s_{0}\right\rangle-\left\langle\mu_{\mathrm{Co}}, y\right\rangle-\lambda\langle 1, y\rangle \\
& =\langle p, y\rangle-\left\langle\lambda-\mu_{\mathrm{St}}(\cdot, T], y\right\rangle-s_{0} \mu_{\mathrm{St}}[0, T]-\left\langle\mu_{\mathrm{Co}}, y\right\rangle
\end{aligned}
$$

since $I_{0}^{*} \mu_{\mathrm{St}}=\mu_{\mathrm{St}}(\cdot, T]$ by Lemma 11 .

To calculate the dual minimand when $(\kappa, \nu) \geq 0$ (which is a dual constraint, since the minimand is $+\infty$ otherwise), note that

$$
\sup _{\left(y, s_{0}\right)} \mathcal{L}^{\mathrm{PS}}=V^{\mathrm{PS}}+\sup _{\left(y, s_{0}\right)} \Pi_{\mathrm{Exc}}^{\mathrm{PS}}
$$

since $V$ is independent of $\left(y, s_{0}\right)$. By (A.6), $\Pi_{\mathrm{Exc}}$ is linear in these variables, so its supremum is either 0 or $+\infty$; and it is zero if and only if $\partial \Pi_{\mathrm{Exc}} / \partial s_{0}=0$ and $\nabla_{y} \Pi_{\mathrm{Exc}}=0$. These conditions are equivalent to (5.5)-(5.6). So the dual programme is: given $(p, k)$, minimise the $V(\kappa, k)$ of (A.4) over $(\kappa, \nu) \geq 0$ and $\lambda$, subject to $(5.5)-(5.6)$.

Comment: In (A.4)-(A.6), $V$ is the value of the available resources $k$, priced at $\kappa$. And $\Pi_{E x c}$ is, for an entrepreneur buying all the inputs, the excess profit (a.k.a. pure profit) from a storage policy $y$ and the use of an initial stock $s_{0}$ : the sum (A.6) defines $\Pi_{\mathrm{Exc}}$ as the total over the cycle of the revenue minus the cost of the resources needed 
at any time $t$. The resources in question are: the time-varying minimum requirements for the capacities (priced at $\kappa$ ), for the stock floor (priced at $\nu_{\mathrm{St}}$ ) and for the required top-up (priced at $\lambda$ ). To see this, recall that $s_{0}-I_{0} y$ is the stock trajectory.

Proof of Remark 2. If this were false, then the minimand's value could be decreased by replacing $\left(\kappa_{\mathrm{St}}, \nu_{\mathrm{St}}, \kappa_{\mathrm{Tu}}, \kappa_{\mathrm{Pu}}\right)$ with $\left(\mu_{\mathrm{St}}^{+}, \mu_{\mathrm{St}}^{-}, \mu_{\mathrm{Co}}^{+}, \mu_{\mathrm{Co}}^{-}\right)$defined by (A.5).

Proof of Proposition 3 (Dual solubility and optimality conditions). Like that of Theorem 1, this proof is put in CP terms. Consider first the dual problem with $L^{\infty *}$, instead of $L^{1}$, as the range for $\kappa_{\mathrm{Pu}}$ and $\kappa_{\mathrm{Tu}}$ in (5.3). Since the nonnegative cones in the (primal) parameter spaces $\left(\mathcal{C}_{+}\right.$and $\left.L_{+}^{\infty}\right)$ have nonempty interiors (for the supremum norm), the framework of [22, Examples 4, 4', 4"] is applicable. To verify the Generalised Slater's Condition of $[22,(8.12)]$ for the primal constraints (4.6)-(4.8), it suffices to take $y=0$, setting $s_{0}$ at any value strictly between 0 and $k_{\mathrm{St}}$. So the dual has a (proper) solution with $\kappa_{\mathrm{Pu}}$ and $\kappa_{\mathrm{Tu}}$ in $L^{\infty *}$, and the primal and dual values are equal (and finite): see, e.g., [22, Theorems 18 (a) and 17 (a)].

To complete the proof of Part 1 it remains to show that $\kappa_{\mathrm{Pu}}$ and $\kappa_{\mathrm{Tu}}$ are in $L^{1}$. From the Hewitt-Yosida decomposition of $(5.6), \kappa_{\mathrm{Tu}}^{\mathrm{FA}}-\kappa_{\mathrm{Pu}}^{\mathrm{FA}}=p_{\mathrm{FA}}=0$ (where $p_{\mathrm{FA}}$ means the purely finitely additive part of $p)$ : see, e.g., [3, Appendix I: (26)-(27)]. Given (5.7), this means that $\kappa_{\mathrm{Tu}}^{\mathrm{FA}}=0=\kappa_{\mathrm{Pu}}^{\mathrm{FA}}$, as required.

For Part 2, apply the Kuhn-Tucker saddle-point characterisation of optima - given in, e.g., [22, Theorem 1 (e) and (f)] - to the primal (4.4)-(4.8) and its dual (5.1)-(5.6). This shows that $\left(y, s_{0}\right)$ and $(\kappa, \nu, \lambda)$ form a dual pair of solutions if and only if they maximise and minimise (respectively) the Lagrange function $\mathcal{L}$ given by (A.3). The minimum in question is characterised by: nonnegativity (5.4), primal feasibility (4.6)(4.8), and complementary slackness, which translates here into Conditions $2 \mathrm{~b}-2 \mathrm{c}$. As for the maximum in question, it is characterised by the conditions $\partial \Pi_{\mathrm{Exc}} / \partial s_{0}=0$ and $\nabla_{y} \Pi_{\mathrm{Exc}}=0$, i.e., $(5.5)-(5.6)$.

Comment: The existence of a dual optimum in the norm-dual spaces $\left(\kappa_{\mathrm{St}}\right.$ and $\nu_{\mathrm{St}}$ in $\mathcal{M}=\mathcal{C}^{*}$, and $\kappa_{\mathrm{Tu}}$ and $\kappa_{\mathrm{Pu}}$ in $L^{\infty *}$ ) comes automatically from (4.11), which ensures that the Generalised Slater's Condition of $[22,(8.12)]$ holds for the norm topologies of the primal parameter spaces $L^{\infty}$ and $\mathcal{C}$. The density representation, of some dual variables, comes from the problem's structure and the assumptions on $p$ : with $p \geq 0$, the optimal $\kappa_{\mathrm{Pu}}$ is in $L^{1}$; and if $p \in L^{1}$ then the optimal $\kappa_{\mathrm{Tu}}$ and $\kappa_{\mathrm{Pu}}$ are both in $L^{1}$.

Proof of Proposition 4 (Stock pricing as the dual). This is a reformulation of Theorem 1: substitute the $\psi$ given by (7.1) into (5.6), and note that, given $\psi$ (and $p$ ), the best choices for $\kappa_{\mathrm{Tu}}$ and $\kappa_{\mathrm{Pu}}$ are $(p-\psi)^{ \pm}$as in (7.4), because $k_{\mathrm{Co}}>0$. This reduces the dual programme (5.1)-(5.6) to minimisation of $k_{\mathrm{St}} \int_{[0, T]}(\mathrm{d} \psi)^{+}+k_{\mathrm{Co}} \int_{0}^{T}|p(t)-\psi(t)| \mathrm{d} t$ over $\psi \in \mathrm{BV}[0-, T+]$, subject to $\psi(0-)=\psi(T+)$ lying between $\psi(0+)$ and $\psi(T-)$. Hence the first of the integrals equals the sum of $(\psi(0+)-\psi(T-))^{+}$and $\int_{(0, T)}(\mathrm{d} \psi)^{+}$; and this sum is $\operatorname{Var}_{\mathrm{c}}^{+}(\psi)$. 
Proof of Proposition 5 (Primal solubility). With $p \in L^{1}$, the maximand of (4.5) is continuous for the weak ${ }^{*}$ topology $\mathrm{w}\left(L^{\infty}, L^{1}\right)$. The feasible set is bounded: in $y$ by (4.6), and in $s_{0}$ by (4.8) with, e.g., $t=0$. So, being also weakly* closed, the feasible set is weakly* compact by the Banach-Alaoglu Theorem. And it is nonempty, since the point $(0,0)$ is feasible by assumption. So an optimum exists by Weierstrass's Extreme Value Theorem.

It is useful to introduce a notation for the sets of those times when the reservoir is empty or full or neither. The sets (which have already appeared in Condition $2 \mathrm{~b}$ of Proposition 3) are:

$$
\begin{aligned}
E(y) & :=\{t \in[0, T]: s(t)=0\} \\
F\left(y, k_{\mathrm{St}}\right) & :=\left\{t \in[0, T]: s(t)=k_{\mathrm{St}}\right\} \\
B\left(y, k_{\mathrm{St}}\right) & :=[0, T] \backslash(E \cup F)=\left\{t: 0<s(t)<k_{\mathrm{St}}\right\},
\end{aligned}
$$

where $y$ is a storage policy (meeting the balance constraint $\left.\int_{0}^{T} y(t) \mathrm{d} t=0\right), s(t)$ is given by (4.9)-(4.10), and $k_{\mathrm{St}} \geq \operatorname{Max}(s)$. Since $s(0)=s(T), 0$ and $T$ are either both in $B$, or both in $E$, or both in $F$. From (4.9), $E \neq \emptyset$. Unless there is spare reservoir capacity, $F \neq \emptyset$ also; and then all the three sets are nonempty. Their connected components are subintervals of $[0, T]$; and, being open, $B$ is the union of a countable (finite or denumerable) sequence of intervals. Those not containing 0 or $T$ are denoted by

$$
A_{m}=\left(\underline{t}_{m}, \bar{t}_{m}\right) \neq \emptyset
$$

for $m=1, \ldots, M \leq \infty$, where $0 \leq \underline{t}_{m}<\bar{t}_{m} \leq T$. If $\{0, T\} \subseteq B$, then $B$ additionally contains two subintervals whose union is

$$
A_{0}=\left(\underline{t}_{0}, T\right] \cup\left[0, \bar{t}_{0}\right)
$$

for some $0<\bar{t}_{0}<\underline{t}_{0}<T$. When $0, T \notin B$, we set for completeness $\underline{t}_{0}=T$ and $\bar{t}_{0}=0$, so that $A_{0}=\emptyset$ in this case. In either case $B=\bigcup_{m>0} A_{m}$.

All these sets may be thought of as subsets of the circle that results from "gluing" 0 and $T$ into a single point $T 0$. Then $\left(A_{m}\right)_{m>0}$ are the component $\operatorname{arcs}$ of $B ; A_{0}$ is that arc which contains $T 0$ (if $T 0 \in B$ ); and $\underline{t}_{m}$ and $\bar{t}_{m}$ are the beginning and the end of $\operatorname{arc} A_{m}$ (w.r.t. the "clockwise" orientation).

The formula for the output $y$, in terms of any $\psi \in \hat{\Psi}$, is proved next. On $\{t: p \neq \psi\}$, the optimal $y$ equals unambiguously $k_{\mathrm{Co}}$ or $-k_{\mathrm{Co}}$. Uniqueness of $y$ on $\{p=\psi\}$ comes from the no-plateau assumption (8.1) on $p$ : this ensures that $\{p=\psi\} \subseteq E \cup F$, up to a null set. And at a.e. $t \in E \cup F$ one has $y(t)=-\dot{s}(t)=0$, since, roughly speaking, $s=$ const. "around" $t$. This argument requires, however, a lemma to remove a technical difficulty that arises because $\left(A_{m}\right)$ can be an infinite sequenceand then the set of component intervals of $F$ and/or $E$ can contain uncountably many singletons, in addition to a countable set of "proper" intervals of positive length. On the interior of such an interval, $\dot{s}=0$ obviously; but this must also be shown to hold 
a.e. on the set of all singleton components of $F \cup E$. And the singletons in question can form a set of positive measure: indeed, all of $F \cup E$ can be a "fat" Cantor-like set that has a positive measure but contains no proper interval.

Lemma 12. If $s$ is a Lipschitz function on $[0, T]$, then $\dot{s}=0$ a.e. on the set $E:=$ $\{t \in[0, T]: s(t)=0\}$.

Proof. See [9].

Proof of Proposition 6 (PS output with plateau-less price). Take any $y \in \hat{Y}$ (not yet known to be unique) and any $\psi \in \hat{\Psi}$ (which may be nonunique, unless $p \in \mathcal{C}$ ). The first and the third lines of (8.2) follow from Part $2 \mathrm{c}$ of Proposition 3 with (5.6) and (7.1). It remains to show that $y=0$ a.e. on the set $S:=\{t: p(t)=\psi(t)\}$. For each $m$, one has $\psi=$ const. on $A_{m}\left(y, k_{\mathrm{St}}\right)$ by Part $2 \mathrm{~b}$ of Proposition 3. Therefore meas $\left(S \cap A_{m}\right)=0$ by $(8.1)$, and hence meas $\left(S \cap B\left(y, k_{\mathrm{St}}\right)\right)=0$ by countable additivity. This means that $S$ is, up to a null set, contained in the set $F\left(y, k_{\mathrm{St}}\right) \cup E(y)$, on which $y=-\dot{s}=0$ a.e. by Lemma 12. This completes the proof of (8.2). It follows that $\hat{Y}$ is a singleton, even when $\hat{\Psi}$ is not. (Given any $\psi \in \hat{\Psi}$, any $y^{\prime}$ and $y^{\prime \prime}$ from $\hat{Y}$ satisfy (8.2) and are therefore equal.)

Proof of Corollary 7 (Dual calculation of SR profit). Formula (9.1) follows from Propositions 3 and 4 . To derive it term-by-term, use the optimality conditions (complementary slackness and feasibility) to expand $\langle p, y\rangle$ :

$$
\Pi:=\int_{0}^{T}(p-\psi) y \mathrm{~d} t+\int_{0}^{T} \psi y \mathrm{~d} t=k_{\mathrm{Co}} \int_{0}^{T}|p-\psi| \mathrm{d} t-\int_{0}^{T} \psi \frac{\mathrm{d} s}{\mathrm{~d} t} \mathrm{~d} t
$$

integrating the last term by parts to obtain

$$
\begin{aligned}
-\int_{0}^{T} \psi \mathrm{d} s & =-[\psi s]_{t=0-}^{t=T+}+\int_{[0, T]} s \mathrm{~d} \psi=s(0)(\psi(0-)-\psi(T+))+k_{\mathrm{St}} \int_{[0, T]}(\mathrm{d} \psi)^{+} \\
& =0+k_{\mathrm{St}} \operatorname{Var}_{\mathrm{c}}^{+}(\psi)
\end{aligned}
$$

as required.

Before a detailed proof of Lemma 8, it is worth presenting the main ideas. The key principle is that a rent can be earned only at a time of full capacity utilisation. In the present context this means that $p-\psi$ can be nonzero only when the converter is working at full power (i.e., when $y(t)= \pm k_{\mathrm{Co}}$ ); and therefore $\psi(t)$ equals $p(t)$ whenever the reservoir is either full or empty (since $s(t)=0$ or $s(t)=k_{\mathrm{St}}$ implies that $\left.y(t)=-\dot{s}(t)=0 \neq \pm k_{\mathrm{Co}}\right)$. By the same principle, $\psi$ can be rising or falling only when the reservoir is full or empty (respectively); so $\psi$ stays constant on each open interval $(\underline{t}, \bar{t})$ during which the reservoir constraints are inactive (i.e., $0<s<$ $\left.k_{\mathrm{St}}\right)$. Together, these conditions determine the function $\psi$ almost completely - except for the possibility of jumps or drops in $\psi$ that may occur at the endpoints of a (closed) interval on which the reservoir is either full throughout or empty throughout. 
Suppose, for example, that $\underline{t}$ is the end of an interval on which the reservoir is full. At that instant, $\psi$ can jump but not drop; and the same is true of $p-\psi$ (since $p=\psi$ just before $\underline{t}$, and $p \geq \psi$ just after $\underline{t}$ ). So neither term, $\psi$ or $p-\psi$, can jump at $\underline{t}$ if their sum $(p)$ is continuous. This determines the constant value of $\psi$ on $(\underline{t}, \bar{t})$ as $p(\underline{t})$; so $\psi$ is unique.

Proof of Lemma 8 (Stock price uniqueness and continuity). Fix any primal solution $y \in \hat{Y}$, which exists by Proposition 5 (though it may be nonunique). To show that there is just one dual solution, we shall express every dual solution $\psi \in \hat{\Psi}$ by the same formula in terms of the fixed $y .{ }^{20}$

In the case of $F\left(y, k_{\mathrm{St}}\right) \neq \emptyset$, which we deal with first, we shall use the Kuhn-Tucker Conditions to show that any $\psi \in \hat{\Psi}$ can be given, in terms of $y$, as

$$
\psi\left(p, k_{\mathrm{PS}}\right)(t)=p(t) \quad \text { for every } t \in(E \cup F)\left(y, k_{\mathrm{St}}\right) \backslash\{0, T\}
$$

whereas on the $m$-th component $A_{m}$ of $B\left(y, k_{\mathrm{St}}\right)$, whose endpoints are $\underline{t}_{m}$ and $\bar{t}_{m}$, it is the constant

$$
\psi\left(p, k_{\mathrm{PS}}\right)(t)=\left\{\begin{array}{ll}
p\left(\underline{t}_{m}\right) & \text { if } \underline{t}_{m} \neq 0 \\
p\left(\bar{t}_{m}\right) & \text { if } \bar{t}_{m} \neq T
\end{array} \quad \text { for every } t \in A_{m}\left(y, k_{\mathrm{St}}\right)\right.
$$

for each $m \geq 0$. Since both $E$ and $F$ are nonempty, $A_{m} \neq(0, T)$, so at least one line of (A.11) applies; and when both do, they are consistent. So (A.10)-(A.11) fully determine $\psi$ on $(0, T)$, and hence on $[0, T]$ because $\psi(0)$ and $\psi(T)$ are defined by continuity.

To use the optimality conditions as stated in Proposition 3 -i.e., in terms of $(\kappa, \nu, \lambda)$ rather than $\psi$-recall from Section 7 that if a $\psi \in \mathrm{BV}(0, T)$ solves $(7.5)-(7.7)$, then (5.1)-(5.6) is solved by: $\left(\kappa_{\mathrm{Tu}}, \kappa_{\mathrm{Pu}}\right)=\left((p-\psi)^{+},(p-\psi)^{-}\right)$, any $\lambda$ between $\psi(0+)$ and $\psi(T-)$, and $\left(\kappa_{\mathrm{St}}, \nu_{\mathrm{St}}\right)=\left(\mu_{\mathrm{St}}^{+}, \mu_{\mathrm{St}}^{-}\right)$, where $\mu_{\mathrm{St}}=\mathrm{d} \psi$ on $(0, T)$ with $\mu\{0\}=\psi(0+)-\lambda$ and $\mu\{T\}=\lambda-\psi(T-)$.

By (5.6),

$$
p=\lambda+\left(\kappa_{\mathrm{St}}-\nu_{\mathrm{St}}\right)[0, \cdot]+\left(\kappa_{\mathrm{Tu}}-\kappa_{\mathrm{Pu}}\right)=\psi+\left(\kappa_{\mathrm{Tu}}-\kappa_{\mathrm{Pu}}\right) \quad \text { a.e. }
$$

It suffices to show that, at every point of $(E \cup F) \backslash\{0, T\}, \psi$ is continuous and equal to $p$ : then (A.11) follows, since $\psi$ is constant on each $B$-component $A_{m}$, and since $A_{m} \neq(0, T)$.

A discontinuity of $\psi$ could only be a jump at a time when the reservoir is full, or a drop when it is empty. If $t \in F$ say, then, being full at $t$, the reservoir cannot be being discharged just before $t$ or charged just after $t .{ }^{21} A$ fortiori, the capacity charge $\kappa_{\mathrm{Tu}}$ must be zero just before $t$, and $\kappa_{\mathrm{Pu}}$ must be zero just after $t$. So $p-\psi=\kappa_{\mathrm{Tu}}-\kappa_{\mathrm{Pu}}$ is

\footnotetext{
${ }^{20}$ The basis for this strategy (used also in proving Proposition 6) is that every dual solution supports every primal solution; i.e., the set of Kuhn-Tucker (saddle) points for a dual pair of convex programmes is the Cartesian product (of the primal and dual solution sets): see Proposition 3.

${ }^{21}$ This, by the way, is where the constancy of $k_{\text {St }}$ over time is used.
} 
nonpositive just before $t$ and nonnegative just after $t$, and hence $p-\psi$ cannot drop at a $t \in F$. This means that any discontinuous changes in $\psi$ and $p-\psi$ are of the same sign and cannot cancel each other out. So $\psi($ and $p-\psi$ ) must be continuous if $p$ is. And it follows (from the signs of $p-\psi$ before and after $t$ ) that $p(t)=\psi(t)$. The "upside down" version of this reasoning applies to $t \in E$.

Since $\kappa_{\mathrm{Tu}}$ and $\kappa_{\mathrm{Pu}}$ are classes of a.e. equal functions (rather than functions), this argument is formalised by using the essential limit concept - for which see, e.g., [4, IV.36-IV.37]. It is also convenient to say that an inequality between functions (of $t$ ) holds somewhere on $A \subseteq[0, T]$ to mean that it holds on an $A^{\prime} \subseteq A$ with meas $A^{\prime}>0$ (i.e., it is not the case that the reverse inequality holds a.e. on $A$ ).

Recall from Section 4 that $y$ with the $\underline{s}_{0, y}$ of (4.9) solve (4.4)-(4.8). Consider first a $t \in F \backslash\{0, T\}$. For every $\Delta t>0$, it cannot be that $y>0$ a.e. on $(t-\Delta t, t)$; i.e., somewhere on $(t-\Delta t, t)$ one has $y \leq 0<k_{\mathrm{Co}}$. Therefore $\kappa_{\mathrm{Tu}}=0$ somewhere on $(t-\Delta t, t)$, by Part 2c of Proposition 3; and, as $\Delta t \rightarrow 0$, this shows that the lower left essential limit of $\kappa_{\text {Tu }}$ at $t$ is zero. Similarly, somewhere on $(t, t+\Delta t)$ one has $y \geq 0>-k_{\mathrm{Co}}$. Therefore $\kappa_{\mathrm{Pu}}=0$ somewhere on $(t, t+\Delta t)$. This means that the lower right essential limit of $\kappa_{\mathrm{Pu}}$ at $t$ is zero; i.e.,

$$
\operatorname{ess} \liminf _{\tau \searrow t} \kappa_{\mathrm{Pu}}(\tau)=0=\operatorname{ess} \liminf _{\tau / t} \kappa_{\mathrm{Tu}}(\tau) \quad \text { for } t \in F \backslash\{0, T\} .
$$

Given (A.12) as well as continuity of $p$ and nonnegativity of $\kappa_{\mathrm{Pu}}$ and $\kappa_{\mathrm{Tu}}$, it follows from (A.13) that ${ }^{22}$

$$
p(t)-\psi(t-)=\operatorname{ess} \lim _{\tau \nearrow t}\left(\kappa_{\mathrm{Tu}}-\kappa_{\mathrm{Pu}}\right)(\tau)
$$

$$
\begin{aligned}
& =\operatorname{ess} \liminf _{\tau \nearrow t} \kappa_{\mathrm{Tu}}(\tau)-\operatorname{ess} \liminf _{\tau \nearrow t} \kappa_{\mathrm{Pu}}(\tau) \leq 0 \\
& \leq \operatorname{ess} \liminf _{\tau \searrow t} \kappa_{\mathrm{Tu}}(\tau)-\operatorname{ess} \liminf _{\tau \searrow t} \kappa_{\mathrm{Pu}}(\tau)=\operatorname{ess} \lim _{\tau \searrow t}\left(\kappa_{\mathrm{Tu}}-\kappa_{\mathrm{Pu}}\right)(\tau) \\
& =p(t)-\psi(t+) .
\end{aligned}
$$

Therefore $\psi(t-) \geq \psi(t+)$ from a comparison of the first and the last sums. But also, since $t \in F$,

$$
\psi(t-) \leq \psi(t+)
$$

by Part 2b of Proposition 3; so all three inequalities of (A.14), (A.15) and (A.16) must actually hold as equalities. This shows that $\psi(t-)=\psi(t+)=p(t)$, i.e., the two-sided limit of $\psi$ at $t$ exists and equals $p(t)$. (Since it exists, it also equals $\psi(t)$ because $\psi(t)$ always lies between $\psi(t-)$ and $\psi(t+)$.) The same can be shown for $t \in E$ (by an "upside down" version of the preceding proof for $t \in F$ ); so

$$
\psi(t)=\lim _{\tau \rightarrow t} \psi(\tau)=p(t) \quad \text { for } t \in(E \cup F) \backslash\{0, T\} \neq \emptyset .
$$

\footnotetext{
${ }^{22}$ This argument uses also the fact that $\lim \inf (A-B) \leq \liminf A-\liminf B \leq \lim \sup (A-B)$ whenever the middle term is well defined. It equals $\lim (A-B)$ if the latter exists, as here (although the inequalities suffice). The same holds with $\lim \sup A-\lim \sup B$ as the middle term.
} 
Nonemptiness of this set follows from the assumption that $F \neq \emptyset$, since $E \neq \emptyset$ always, by (4.9).

By Part $2 \mathrm{~b}$ of Proposition $3, \psi$ is constant on each $A_{m}$. This and (A.17) show that $\psi \in \mathcal{C}(0, T)$. (Equivalently $\psi \in \mathcal{C}[0, T]$, since $\psi(0):=\psi(0+)$ and $\psi(T):=\psi(T-)$.)

It remains to check that the proven properties of $\psi$ imply (A.11). Since $E \cup F \nsubseteq$ $\{0, T\}$, the set $B$ consists of two or more nonempty components $A_{m}$. Each of these has at least one endpoint that is neither 0 nor $T$; i.e., $\underline{t}_{m} \neq 0$ or $\bar{t}_{m} \neq T\left(\underline{t}_{m} \neq T\right.$ and $\bar{t}_{m} \neq 0$ always). Say it is $\underline{t}_{m}$; then $\underline{t}_{m} \in(E \cup F) \backslash\{0, T\}$, since $\underline{t}_{m} \notin A_{m}\left(A_{m}\right.$ is an open arc). So, by (A.17) and the constancy of $\psi$ on $A_{m}$,

$$
p\left(\underline{t}_{m}\right)=\psi\left(\underline{t}_{m}\right)=\psi(t) \quad \text { for every } t \in A_{m} .
$$

If $T \neq \bar{t}_{m}$, then (A.18) holds with $\bar{t}_{m}$ in place of $\underline{t}_{m}$, by the same argument. This also shows that $p\left(\underline{t}_{m}\right)=p\left(\bar{t}_{m}\right)$ if both $\underline{t}_{m} \neq 0$ and $\bar{t}_{m} \neq T$. (All this applies to $m=0$ as well, if $A_{0} \neq \emptyset$. In this case $\psi$ is additionally constant on $A_{0} \supset\{0, T\}$; so $\psi(0)=\psi(T)$ even if $p(0) \neq p(T)$.) This completes the proof of (A.10)-(A.11) when $F \neq \emptyset$.

If $p(0)=p(T)$, then $\psi(0)=\psi(T)$ follows by virtually the same argument as that proving (A.17), with 0 and $T$ thought of as a single point of the circle.

Finally, consider the case of $F\left(y, k_{\mathrm{St}}\right)=\emptyset$, which is trivial in that the reservoir is never used to capacity, and it earns no rent. Formally, $\kappa_{\mathrm{St}}=\nu_{\mathrm{St}}=0$ by Part $2 \mathrm{~b}$ of Proposition 3 and (5.5); so $\psi$ is a constant. Its uniqueness is readily shown: $\psi$ minimises (7.6) over BV $(0, T)$, so, a fortiori, it minimises (7.6) over $\mathbb{R}$. Since for $\psi \in \mathbb{R}$ the sum (7.6) simplifies to $k_{\mathrm{Co}} \int_{0}^{T}|p(t)-\psi| \mathrm{d} t$, the minimum in question is characterised by the FOC

$$
\text { meas }\{t: p(t)<\psi\} \leq \frac{T}{2} \leq \operatorname{meas}\{t: p(t) \leq \psi\},
$$

which means that $\psi$ is a median for the distribution of $p$ w.r.t. meas $/ T$. And the median is unique if $p \in \mathcal{C}[0, T]$, since the cumulative distribution function of $p$ is then strictly increasing on the interval $(\operatorname{Min}(p), \operatorname{Max}(p))$.

Comment: Although (A.13) suffices for the argument, both inf signs can be deleted, i.e., (A.13) can be strengthened to: $\kappa_{\mathrm{Tu}}(t-)=0=\kappa_{\mathrm{Pu}}(t+)$ with $\kappa_{\mathrm{Tu}}(t+) \geq 0$ and $\kappa_{\mathrm{Pu}}(t-) \geq 0$, for $t \in F \backslash\{0, T\}$, whenever $p(t \pm)$ exist. This is because, by (5.7) and the continuity of $\kappa \mapsto \kappa_{ \pm} \in \mathbb{R}_{+}$, the four limits exist and are equal to $\left(\kappa_{\mathrm{Tu}}-\kappa_{\mathrm{Pu}}\right)_{ \pm}(t \pm)=(p-\psi)_{ \pm}(t \pm)$. All four limits are zero if $p$ is continuous at $t$.

Before a formal proof of Theorem 9 , it is worth retracing in the present context the familiar argument which establishes the derivative property of the value function when differentiability is taken for granted. With the dual minimand (7.6) denoted by $V\left(k_{\mathrm{PS}}, \psi\right)$, the r.h.s.'s of (9.3)-(9.4) are obviously the partial derivatives of $V$ in $k_{\mathrm{PS}}$, evaluated at the dual optimum $\hat{\psi}\left(k_{\mathrm{PS}}\right)$. And the total derivatives, in $k_{\mathrm{PS}}$, of the dual value $V\left(k_{\mathrm{PS}}, \hat{\psi}\left(k_{\mathrm{PS}}\right)\right)$ are equal to the corresponding partial derivatives, since the 
partial derivative of $V$ in $\psi$ vanishes by the FOC for optimality of $\hat{\psi}$. To complete the calculation, note that the dual value equals the primal value $\Pi_{\mathrm{SR}}^{\mathrm{PS}}$ (if $k_{\mathrm{PS}} \gg 0$ ). This is, indeed, the substance of the first step in the Proof of Theorem 9, except that a standard convex duality result is used instead of the above derivation "from first principles". This is necessary because a rigorous application of the chain rule would run into difficulties, since it would require the differentiability of $\hat{\psi}$ in $k_{\mathrm{PS}}$, and of $V$ in $\psi$. This would make their composition $\Pi\left(k_{\mathrm{PS}}\right)=V\left(k_{\mathrm{PS}}, \hat{\psi}\left(k_{\mathrm{PS}}\right)\right)$ differentiable, but neither this nor even the uniqueness of an optimal $\psi$ (i.e., the existence of $\hat{\psi}$ ) may be presupposed. Rather, these properties must be derived - by using price continuity, since they are known to fail in general if $p \notin \mathcal{C}$ (Example 10). This gap is filled by Lemma 8.

Proof of Theorem 9 (Efficiency rents of a storage plant). The first, routine, step is to identify the dual variables as marginal values of the primal parameters, with the marginal values formalised as supergradients (of the primal value, a concave function of the parameters): see, e.g., [22, Theorem 16: (b) and (a), with Theorem 15: (e) and (f)] or [17, 7.3: Theorem 1']. This is applied in such a way as to give the marginal interpretation to the optimal $\kappa$ and $\nu$ themselves, rather than only to their totals over the cycle, although the formulae to be proved are for the total values. Therefore the SR profit is considered as a function, $\tilde{\Pi}_{\mathrm{SR}}^{\mathrm{PS}}$, of all the quantity parameters

$$
\left(k_{\mathrm{St}}, n_{\mathrm{St}}, k_{\mathrm{Tu}}, k_{\mathrm{Pu}}, \zeta\right) \in \mathcal{C} \times \mathcal{C} \times L^{\infty} \times L^{\infty} \times \mathbb{R}
$$

discussed in Section 5. It is an extension of the optimal value of the programme (4.4)-(4.8), i.e.,

$$
\Pi_{\mathrm{SR}}^{\mathrm{PS}}\left(p ; k_{\mathrm{St}}, k_{\mathrm{Co}}\right)=\tilde{\Pi}_{\mathrm{SR}}^{\mathrm{PS}}\left(p ; k_{\mathrm{St}}, 0, k_{\mathrm{Co}}, k_{\mathrm{Co}}, 0\right) \quad \text { for }\left(k_{\mathrm{St}}, k_{\mathrm{Co}}\right) \in \mathbb{R}^{2},
$$

where the scalars are identified with constant functions on $[0, T]$. In this setting, the result giving the marginal values of the primal parameters is

$$
\begin{aligned}
\partial_{k_{\mathrm{St}}, n_{\mathrm{St}}, k_{\mathrm{Tu}}, k_{\mathrm{Pu}}, \zeta} \tilde{\Pi}_{\mathrm{SR}}^{\mathrm{PS}=} & \left\{\left(\kappa_{\mathrm{St}},-\nu_{\mathrm{St}}, \kappa_{\mathrm{Tu}}, \kappa_{\mathrm{Pu}}, \lambda\right):(\kappa, \nu, \lambda)\right. \text { meet } \\
& \text { Conditions } 2 \mathrm{a}, 2 \mathrm{~b} \text { and } 2 \mathrm{c} \text { of Proposition } 3\} .
\end{aligned}
$$

For differentiation of $\Pi_{\mathrm{SR}}^{\mathrm{PS}}$, with respect to the constant capacities, it follows from (A.20) that

$\partial_{k_{\mathrm{St}}, k_{\mathrm{Co}}} \Pi_{\mathrm{SR}}^{\mathrm{PS}}=\left\{\left(\int_{[0, T]} \kappa_{\mathrm{St}}(\mathrm{d} t), \int_{0}^{T}\left(\kappa_{\mathrm{Tu}}+\kappa_{\mathrm{Pu}}\right)(t) \mathrm{d} t\right): \exists \nu \exists \lambda(\kappa,-\nu, \lambda) \in \partial_{k_{\mathrm{PS}}, n_{\mathrm{St}}, \varsigma} \tilde{\Pi}_{\mathrm{SR}}^{\mathrm{PS}}\right\}$

$$
=\left\{\left(\operatorname{Var}_{\mathrm{c}}^{+}(\psi), \int_{0}^{T}|p(t)-\psi(t)| \mathrm{d} t\right): \psi \in \hat{\Psi}\left(p ; k_{\mathrm{St}}, k_{\mathrm{Co}}\right)\right\},
$$

by using (7.4) and substituting $\kappa_{\mathrm{St}}=(\mathrm{d} \psi)^{+}$. When $p \in \mathcal{C}$, the set $\hat{\Psi}$ is actually a singleton by Lemma 8 , and hence so is $\partial_{k_{\mathrm{PS}}} \Pi_{\mathrm{SR}}^{\mathrm{PS}}$. 



\section{REFERENCES}

[1] Anderson, E. J., and P. Nash (1987): Linear programming in infinite-dimensional spaces. New York-Chichester-Brisbane-Toronto-Singapore: Wiley.

[2] Bauer, W., H. Gfrerer, and H. Wacker (1984): "Optimization strategies for hydro energy storage plants", Zeitschrift für Operations Research, Series B, 28, 103-131.

[3] Bewley, T. (1972): "Existence of equilibria in economies with infinitely many commodities", Journal of Economic Theory, 4, 514-540.

[4] Dellacherie, C., and P. A. Meyer (1978): Probabilities and potential. Amsterdam-New YorkOxford: North-Holland.

[5] Foran, J. (1991): Fundamentals of real analysis. New York-Basel-Hong Kong: Dekker.

[6] Gravelle, H. S. E. (1976): "The peak-load problem with feasible storage", Economic Journal, $86,256-277$.

[7] Horsley, A., and A. J. Wrobel (1986): "The Mackey continuity of the monotone rearrangement", Proceedings of the American Mathematical Society, 97, 626-628.

[8] Horsley, A., and A. J. Wrobel (1988): "Subdifferentials of convex symmetric functions: An application of the Inequality of Hardy, Littlewood and Pólya", Journal of Mathematical Analysis and Applications, 135, 462-475.

[9] Horsley, A., and A. J. Wrobel (1996): "Efficiency rents of storage plants in peak-load pricing, I: pumped storage", STICERD Discussion Paper TE/96/301, LSE.

[10] Horsley, A., T. Van Zandt, and A. J. Wrobel (1998): "Berge's Maximum Theorem with two topologies on the action set", Economics Letters, 61, 285-291.

[11] Horsley, A., and A. J. Wrobel (1999): "Boiteux's solution to the shifting-peak problem and the density form of equilibrium prices in continuous time", STICERD Discussion Paper TE/99/371, LSE (submitted to Economic Theory).

[12] Horsley, A., and A. J. Wrobel (1999): "Efficiency rents of storage plants in peak-load pricing, II: hydroelectricity", STICERD Discussion Paper TE/99/372, LSE.

[13] Horsley, A., and A. J. Wrobel (1999): "Efficiency rents of hydroelectric storage plants in continuous-time peak-load pricing", in The Current State of Economic Science (Volume 1, pp. 453-480), ed. by S. B. Dahiya. Spellbound Publications: Rohtak.

[14] Horsley, A., and A. J. Wrobel (2000): "Localisation of continuity to bounded sets for nonmetrisable vector topologies and its applications to economic equilibrium theory", Indagationes Mathematicae (New Series), 11, 53-61.

[15] Horsley, A., and A. J. Wrobel (2000): "The short-run approach to LRMC pricing for multiple outputs with nondifferentiable costs", STICERD Discussion Paper TE/00/393, LSE (submitted to Journal of Economic Theory).

[16] Horsley, A., and A. J. Wrobel (2000): "Continuity of the equilibrium price density, with an application to peak-load pricing with storage", a forthcoming STICERD Discussion Paper, LSE.

[17] Ioffe, A. D., and V. M. Tihomirov (1979): Theory of extremal problems. Amsterdam-New York-Oxford: North-Holland.

[18] Koopmans, T. C. (1957): "Water storage policy in a simplified hydroelectric system", in: Proceedings of the First International Conference on Operational Research, pp. 193-227. LondonBaltimore. (Cowles Foundation Paper No. 115. Also in: The collected papers of T. C. Koopmans, pp. 282-316.)

[19] Moore, T. (1994): "Storing megawatthours with SMES", Electric Power Research Institute Journal, vol. 19, No. 5, 24-33.

[20] Nguyen, D. T. (1976): "The problems of peak loads and inventories", Bell Journal of Economics, 7, 232-241.

[21] Pyatt, G. (1978): "Marginal costs, prices and storage", Economic Journal, 88, 749-762. 
[22] Rockafellar, R. T. (1974): Conjugate duality and optimization. Philadelphia, PA: SIAM.

(Anthony Horsley and Andrew J. Wrobel) Department of Economics, London School of Economics, Houghton Street, London WC2A 2AE, United Kingdom

E-mail address: LSEecon123@msn.com 